\title{
Framework Confirmation by Newtonian Abduction ${ }^{\dagger}$
}

\author{
Erik Curiel ${ }^{\ddagger}$
}

\begin{abstract}
The analysis of theory-confirmation generally takes the deductive form: show that a theory in conjunction with physical data and auxiliary hypotheses yield a prediction about phenomena; verify the prediction; provide a quantitative measure of the degree of theory-confirmation this yields. The issue of confirmation for an entire framework (e.g., Newtonian mechanics en bloc, as opposed, say, to Newton's theory of gravitation) either does not arise, or is dismissed in so far as frameworks are thought not to be the kind of thing that admits scientific confirmation. I argue that there is another form of scientific reasoning that has not received philosophical attention, what I call Newtonian abduction, that does provide confirmation for frameworks as a whole, and does so in two novel ways. (In particular, Newtonian abduction is not inference to the best explanation, but rather is closer to Peirce's original idea of abduction.) I further argue that Newtonian abduction is at least as important a form of reasoning in science as standard deductive and inductive forms. The form is beautifully summed up by Maxwell (1876): "The true method of physical reasoning is to begin with the phenomena and to deduce the forces from them by a direct application of the equations of motion."
\end{abstract}

\section{Contents}

1 Types of Reasoning in Science

2 Frameworks

${ }^{\dagger}$ This paper is forthcoming in Synthese, 2019 (doi:10.1007/s11229-019-02400-9). I thank Bill Harper for many enjoyable and edifying discussions about Newton, scientific reasoning, and evidence, and for having written such a wonderful book (Harper 2011). I also thank him for detailed comments on an earlier draft of the paper, including catching an error in $\S 3$ in my discussion of Hall, Brown, and the precessions of Mercury and the Moon. I thank Tom Pashby for comments on an earlier draft, including interesting suggestions for elaboration in future work. I thank an anonymous referee for detailed criticisms and questions. I am grateful to Howard Stein as well, as always, for many illuminating and pleasurable conversations on all of these matters. Work for this paper was funded by grant CU 338/1-1 from the Deutsche Forschungsgemeinschaft.

${ }^{\ddagger}$ Author’s address: Munich Center for Mathematical Philosophy, Ludwig-Maximilians-Universität; Black Hole Initiative, Harvard University; Smithsonian Astrophysical Observatory, Radio and Geoastronomy Division; email: erik@strangebeautiful.com 
5 This Is True Confirmation

References

For the whole difficulty of [natural] philosophy seems to be to discover the forces of nature from the phenomena of motions and then to demonstrate the other phenomena from these forces.

Newton

Principia, "Preface"

\section{Types of Reasoning in Science}

In so far as one can identify something like a "standard view" in the philosophical debates over a broad and vague topic like confirmation, and in so far as one can make precise the distinction between frameworks and theories, it is the standard view in the philosophical community that frameworks are such things as not to admit confirmation. Friedman (2001), for instance, in his influential discussion of frameworks and how what he calls their relativized a priori principles allow theory to make substantive contact with experimental data, explicitly argues that frameworks, based on the foundational roles they play in evidentiary reasoning, cannot admit confirmation in any standard sense on pain of circularity. Based on his view of the role frameworks play in grounding the meaning of terms in theories and the possibility of applying theories to reason about phenomena, Carnap (1956) was at pains to emphasize that the choice of framework is just that, a choice, a practical action and not the assertion of a proposition whose possible truth-value or doxastic support can be evaluated in rationally principled ways; as such, the issue of confirmation, which he took to be expressible by a proposition with determinate cognitive content, whose truth value could be objectively evaluated, did not arise with regard to frameworks. Kuhn (1996) is perhaps the locus classicus of such a view, with his extravagant claim of the fundamental and incorrigible irrationality in the acceptance and use of a framework (or "paradigm"). ${ }^{1}$

In this paper, I argue to the contrary. There is a form of scientific reasoning that has not received philosophical attention, which I call Newtonian abduction, whose successful application provides direct and strong confirmatory support for frameworks as a whole, not just for theories that one may formulate in the context of the framework. The two forms of confirmation that accrue to it, what I call structural confirmation and modal confirmation, have not to my knowledge been characterized in the literature, and are of interest in themselves.

\footnotetext{
${ }^{1}$ Those who advocate some form of holism about knowledge will also hold that frameworks admit of confirmation. Quine, the canonical example of a holist, thought that frameworks admit empirical confirmation because he thought everything does, even pure mathematics. My reasons for claiming that frameworks admit confirmation differ from those of the holist: as will become clear in the paper's argument, I claim they do only in so far as the representations they allow us to construct make substantive, direct contact with the physical systems they purport to treat. That is certainly not true of pure mathematics qua mathematics. It is also the case that some advocates of inference to the best explanation (IBE), such as Lipton (2004), claim that it allows for confirmation of frameworks. See footnote 42 below for a comparison of the ways that advocates of IBE claim that it supports confirmation of frameworks to the ways I argue Newtonian abduction does.
} 
Above and beyond its role in confirmation of frameworks, moreover, I claim this form of reasoning lies at the heart of many of the most important advances in physics since the time of Newton. A detailed defense of that claim is beyond the scope of this paper, but I hope that my explication of it and the examples I adduce and discuss will go a long way towards making that case. In any event, my discussion will show that it is an important and fundamental form of reasoning in physics that is logically, conceptually and methodologically distinct from hypothetico-deductive reasoning, inductive reasoning, and IBE. As such it deserves investigation by philosophers of science, and one can reasonably expect such investigation to offer new illumination on many important problems besides confirmation, such as the nature of scientific explanation and understanding, the character of evidential relations and the kinds of epistemic warrant evidence may provide for theoretical claims, the way that theory and experiment make contact with each other in general, a variety of inter-theoretic relations such as reduction and emergence, and so on.

The standard form of confirmation most treated in the literature is, in effect, that characterized by the following schema:

1. theory + auxiliary hypotheses + initial conditions $\Rightarrow$ prediction;

2. verify prediction;

3. apply qualitative or quantitative measure of degree of confirmation to theory.

Of course, there are many substantive differences among the many different approaches to the study of confirmation (Earman and Salmon 1992; Norton 2005), but for my purposes using this schema as representative of the primary ones suffices. As the basis for philosophical reasoning about confirmation, the schema is not wrong so far as it goes - but it goes only so far. It does not capture the confirmatory potential of other forms of reasoning, and, most important for my purposes, not of Newtonian abduction. ${ }^{2}$

Roughly speaking, Newtonian abduction is the derivation of the equations of motion, within a framework, of a particular type of physical system, based on a given class of evolutions that type of system may manifest: given a framework and a class of observed physical evolutions of a type of system, one deduces from these the unique theory of that type of system as formulated in the framework. Newtonian abduction shows the framework to be capable of accounting for phenomena by providing generic forms of equations of motion that, in the framework's context, are necessary and sufficient for the sound representation of the observed phenomena, and from which further specific predictions can be derived. ${ }^{3}$ It therefore, in a sense, when available, grounds

\footnotetext{
${ }^{2}$ In order to try to avoid misunderstanding, I emphasize that I am not claiming that all forms of reasoning treated by systems, such as the Bayesian one, in which one tries to model confirmataory relations, are subsumed by the schema that I claim represents the "standard form of confirmation". Philosophers have given strong arguments that the Bayesian framework, for example, can model the confirmatory relevance of forms of reasoning such as statistical inference, unification, explanation, and partial entailment. I still think, nonetheless, that the-admittedly grosssimplification of using the logical form of HD as the comparative foil for the structure of confirmation in Newtonian abduction - as I will do throughout the paper - is justified for my purposes, since all the other forms of reasoning that, e.g., Bayesianism treats (unification, explanation, partial entailment, and so on) have the same difficulty capturing the kinds of confirmation that Newtonian abduction endows frameworks with, what I call below structural and modal confirmation.

${ }^{3}$ I will speak of "equations of motion" in this paper, but it should be understood that what I say applies also to field equations, and indeed to any mathematical relations and physical principles a framework or theory posits as governing and constraining the behavior of the types of physical system it treats. I will discuss examples of more
} 
the standard form of confirmation based on deductive and other forms of reasoning, as adumbrated in the schema above.

Before going any further, I must emphasize that Newtonian abduction has nothing to do with IBE, which is these days often referred to as 'abduction'. Although the possibility of confusion is not negligible, I like the name 'Newtonian abduction' for the type of reasoning I characterize for two reasons. First, it was, to the best of my knowledge, first and most consistently and powerfully deployed by Newton. Second, it corresponds closely in logical form - or perhaps logical intent is more to the point - to the idea of abduction as originally promulgated by Peirce (1878a), Peirce (1903), Peirce (1955). ${ }^{4}$ I will briefly explain at the end of $\S 3$, after I explicate and characterize the idea of Newtonian abduction, this similarity of the form and intent, to justify my use of the term. Also Newtonian abduction is not the same as what is known in the literature as Newton's "deduction from the phenomena" (Harper 1990; Worrall 2000), though the two are related in important ways; indeed, as we will see, one may with some justice view the standard account of Newton's "deduction from the phenomena" as capturing part of the full structure of Newtonian abduction.

The rest of the paper is as follows. In $\S 2$, I characterize what I mean by a framework. In $\S 3$, I characterize Newtonian abduction as a form of reasoning within frameworks, and discuss its salient logical and conceptual features. In $\S 4$, I sketch the way that successful Newtonian abduction lends direct and immediate confirmatory support to frameworks, and characterize the kinds of confirmation that accrue to it. I conclude in $\S 5$ by arguing against a possible objection to the claim that Newtonian abduction lends confirmatory support to the framework itself, and by discussing the relation of Newtonian abduction to the idea of understanding in science; I also briefly discuss the disconfirmation of frameworks.

\section{$2 \quad$ Frameworks}

In this section, I explain what I mean by 'framework'. I neither promise nor threaten you with anything near a complete, precise definition. For my purposes, it suffices to provide only a rough characterization sketched in broad strokes. ${ }^{5}$

A framework is a system that allows one to formulate propositions and affirm them in principled ways based on evidence gathered according to good principles and to apply these in turn as evidence based on good principles. So far, that does not differentiate it from a theory. It is so differentiated by the fact that some of the propositions it allows one to formulate are themselves

general such types of relation and principle at the end of $\S 3$.

${ }^{4}$ It seems to be the case that many if not most contemporary philosophers believe that Peirce's notion of abduction is in fact IBE. That is wrong. An attentive reading of Peirce shows that his notion of abduction was not equivalent to contemporary conceptions of IBE, and that neither did he ever champion any such idea. Detailed exegesis to demonstrate this is beyond the scope of this paper. I will only invite the reader to read Peirce (1878a), Peirce (1903), Peirce (1955) with an unbiased eye, and note that nowhere does Peirce use the "goodness" of the hypothesis as grounds for formulating it based on the evidence. He certainly expected that one would, in the natural course of events, evaluate the goodness of the hypothesis once one had produced it by abduction, but the goodness of the hypothesis plays no role for him in its production by abductive reasoning. As I will argue, the same holds true for Newtonian abduction.

${ }^{5}$ I discuss almost all the issues here in greater detail and to greater depth in Curiel (2019). 
theories. ${ }^{6}$ A theory in this sense is a system that allows for the unified representation and modeling of a particular kind of physical system so as to render that kind amenable to investigation by scientific reasoning and practices of all forms. A particular kind of physical system is one such that all individuals falling under the kind bear the same physical quantities whose properties are characterized by and whose behavior is governed by the same set of equations of motion and collateral mathematical relations, such as kinematical constraints (Curiel 2017a). A theory in my sense also includes such things as accounts of experimental devices appropriate for the study of the relevant kind of physical system, good practices for employing them, sound techniques for the collection of raw data and statistical and other analysis and organization of the same, reliable methods of approximative and heuristic reasoning for constructing models and solving equations, and guidelines for determining whether a system of the given kind is in a state and experiencing interactions with its environment such as to be jointly amenable to appropriate and adequate representation by the theory (viz., whether or not the system falls into the theory's regime of applicability, as I will call it), and so on. ${ }^{7}$ These ideas are for my purposes adequately clarified by examples.

Newtonian mechanics, the heart of which is embodied in the definitions and the three laws Newton lays out in his Principia, is a framework. Newton's Second Law in the abstract is not the equation of motion of any particular kind of physical system. It is rather the schema that any equation of motion for any particular kind of system treated by the framework must instantiate. Newton's theory of gravity is a theory formulated in the framework of Newtonian mechanics. It treats that kind of physical system characterized, inter alia, by the possession of inertial mass, gravitational mass, spatial position, and a velocity expressible as the temporal derivative of spatial position, all such that the system's dynamical evolution is governed by Newton's gravitational force law. Navier-Stokes theory, the classical theory of thermoconducive, viscöelastic fluids, is another

\footnotetext{
${ }^{6} \mathrm{My}$ conception of a framework is in many ways similar to, and indeed inspired by, Carnap's conception of a linguistic framework (Carnap 1956), particularly in the way that a framework in both senses serves to define a fixed sense of physical possibility relevant to the kinds of system the framework treats. Carnap's conception is too broad and vague, however, to do the work I require of it. Stein (1992) provides an insightful and illuminating, albeit brief, discussion of the differences between a Carnapian framework in the original sense and a framework in the sense of a structure in theoretical physics of the type I am sketching here, though he restricts attention to the strictly mathematical parts of one, as I do not. Lakatos (1970) has some affinity with the gist of this view, in particular his notion of the "hard core" of "research programs", though, again, the differences in detail outweigh the similarities. There is perhaps more affinity with the "research traditions" of Laudan (1977), in so far as different ones can share and swap important methodological and theoretical principles, as can happen with frameworks in my sense. A discussion of these comparisons is beyond the scope of this paper.

${ }^{7}$ It is also characteristic of an appropriately unified kind of physical system, one treated by a theory in my sense, that there exist a set of scales at each of which all quantities the theory attributes to the kind of system simultaneously lose definition - the breakdown scales - as does not happen with the entirety of the family of all types of physical system treated by a framework. In other words, every theory has a single, unified regime of applicability, bounded on all sides by scales characterized by the values of different combinations of its physical quantities. For classical fluids, for example, the definitions of their pressure, fluid flow, viscosity, and all the rest break down at spatial and temporal scales a few orders of magnitude greater than those of the mean free-path of the fluid's constituent particles. There is no a priori reason why the definitions of all the different physical quantities represented by the theory should fail at the same characteristic scales, even though, in fact, those of all known theories do, not only for classical fluids but for all physical theories we have. This seems, indeed, to be one of the markers of a physical theory, the existence of a set of characteristic scales for its physical quantities, at each of which all the theory's physical quantities simultaneously lose definition. This is a fact that deserves philosophical investigation.
} 
theory formulated in the framework of Newtonian mechanics. It treats that kind of physical system characterized, inter alia, by the possession of inertial mass, shear viscosity, bulk viscosity, thermal conductivity, fluid velocity, heat flux, pressure, and shear-stress, all satisfying among themselves fixed relations of constraint (e.g., that heat flux is always independent of the pressure gradient), and whose dynamical evolution is governed by the Navier-Stokes equations. ${ }^{8}$ One can also formulate Navier-Stokes theory in the framework of Lagrangian mechanics. On the conception I put forward here, special relativity is a framework, and one can formulate in it the theory of classical Maxwell fields, as well as the theory of the mechanics of relativistic point-particles. Non-relativistic quantum mechanics is a framework, within which one can formulate the atomic theory of elements of low atomic weight. Quantum field theory is a framework within which one can formulate the theory of quantum electrodynamics. Somewhat more controversially, I suspect, according to my view general relativity is a framework within which one can formulate theories of dust, fluids, Maxwell fields, Dirac fields, and so on. ${ }^{9}$

My analysis and arguments will need further distinctions with regard to "level" of theoretical representation, so it will be useful to characterize them by laying down terminology. The schema in a framework for equations of motion and other mathematical relations I call abstract. Canonical examples are Newton's Second Law, the Schrödinger equation in non-relativistic quantum mechanics, and so on. Structure and entities at the highest level of a theory formulated in a given framework I will call generic. In particular, generic structure has no definite values for those quantities that appear as constants in the theory's equations of motion and other mathematical relations. The symbol ' $k$ ' appearing in the generic equation of motion of an elastic spring modeled as a simple harmonic oscillator, $\ddot{x}=-\frac{k}{m} x$, denotes Hooke's constant (the coefficient of proportionality between a force applied to the spring and the resulting diplacement from its equilibrium position), but possesses no fixed value, and the same for the mass $m$. It is important to keep in mind, however, that all these formal representations of physical quantities do have determinate physical dimensions, for Hooke's constant, e.g., $\frac{\text { mass }}{\text { time }^{2}}$. Otherwise, one could not say that this is the generic equation of motion for, say, a spring rather than a pendulum or oscillating string or electric circuit or any other type of system whose dynamical evolution is governed by the equation of motion for a simple harmonic oscillator.

One can, in the same way, write down generic solutions to the generic equations of motion. These are formal representations of the dynamically possible evolutions such systems can manifest. One generic solution to the equation of motion of an elastic spring is $x(t)=\cos \left(\sqrt{\frac{k}{m}} t\right)$, where again one may think of ' $k$ ' and ' $m$ ' as dummy variables, not determinate real numbers. Generic structure defines a genus of physical system, that type of physical system the theory appropriately and adequately treats.

One obtains specific structure by fixing the values of all such constants in generic structure,

\footnotetext{
${ }^{8}$ See Landau and Lifschitz $(1975$, ch. v, §49) for an exposition of the physics of Navier-Stokes theory, and Curiel (2017c) for a deeper and more extensive discussion of Navier-Stokes theory with regard to the issues I discuss here. There is a subtlety I am glossing over: Navier-Stokes theory requires partial-differential equations for its formulation, which were not a part of the original Newtonian framework. Strictly speaking, therefore, Navier-Stokes theory is formulated in an appropriate extension of the original Newtonian framework. How frameworks can be extended in such ways is a fascinating problem, but one I cannot discuss here.

${ }^{9} \mathrm{I}$ argue elsewhere for the claim that general relativity is a framework in this sense, and not a theory. Nothing in the paper hinges on the claim, so if you object to it, let it go.
} 
say $m=1$ and $k=5$ (in some system of units) for the elastic spring. This defines a species of physical system of that genus, all springs with those values for mass and Hooke's constant. ${ }^{10}$ One now has a determinate space of states for systems of that species, and a determinate family of dynamically possible evolutions, viz., the solutions to the specific equations of motion, represented by a distinguished family of paths on the space of states. A path in this family is an individual model (or individual solution) of the specific equations of motion; one common way of fixing an individual model is by fixing definite initial conditions for the specific equations of motion. An individual model, as the name suggests, represents a unique physical system of the species, that whose dynamical quantities satisfy the initial conditions. In the case of the spring, that may mean the unique one whose position and momentum at a given time have the values given by the initial conditions. ${ }^{11}$

Finally, I call a concrete model a collection of experimentally or observationally gathered results analyzed, structured and interpreted in such a way as to allow identification with an individual model; I will sometimes also refer to a concrete model as structured data. ${ }^{12}$ If I continually measure the position and momentum of a spring with known values for $m$ and $k$, oscillating in one dimension for an interval of time equal to its period of oscillation, and graph the results "in a natural way", e.g., as a curve parametrized by time on a Cartesian plane whose $x$-axis represents position and $y$-axis represents momentum, then I will produce a curve that can be, "in a natural way", identified with exactly one dynamically possible evolution on the space of states used to represent that species of spring. Kepler's organization and structuring of the planetary ephemerides into parametrized ellipses satisfying the Area Law and the Harmonic Law is an example that I will rely on in some detail in the next section. There are many fascinating and deep problems associated with characterizing the ways that experimental and observational results can be appropriately transformed into structured data and identified with structures in theory, but I put them aside for my purposes here. ${ }^{13}$

\footnotetext{
${ }^{10}$ One can as well consider mixed systems, with, say, a fixed value for mass but indeterminate value for Hooke's constant. These raise interesting questions, but they are beside the point here.

${ }^{11}$ I have deliberately taken my terminology from biological taxonomy, inspired by the remark of Peirce (1878b, p. 143):

Now, the naturalists are the great builders of conceptions; there is no other branch of science where so much of this work is done as in theirs; and we must, to great measure, take them for our teachers in this important part of logic.

There is much of insightful relevance in the lead-up to this remark, about how one individuates and characterizes genera and species of physical systems in my sense, which it would be illuminating to discuss, but it would take us too far afield. I am tempted to describe structure at the level of a framework as phylar, and to call the family of all types of physical system treated by a framework a phylum - and so all physical systems would fall under the kingdom of physics - but I suspect it would just be distracting to the reader.

${ }^{12}$ This idea bears obvious and interesting comparison with the distinction between data and phenomena as drawn by Bogen and Woodward (1988). In so far as I understand their distinction, their idea of data more or less corresponds with my idea of "experimentally or observationally gathered results", but their notion of phenomena, in so far as it seems to try to capture something like general patterns in the world, does not neatly square with my conception of a concrete model, which is the result of appropriately transforming the results of a single experiment (or family of related experiments).

${ }^{13}$ I discuss many of them in detail in Curiel (2019).
} 


\section{Abduction}

I can now give a somewhat precise definition: Newtonian abduction is the demonstration that a theory (generic equations of motion) in a framework is logically equivalent to a concrete model of a system appropriately treated by it. It is the use of observed dynamical evolutions of concrete physical systems to derive from an abstract framework, such as Newtonian mechanics, the form of equations of motion for systems of the given genus, such as Newton's gravitational law. It has the logical form

$$
\text { framework } \Rightarrow \text { (concrete model } \Leftrightarrow \text { generic equations of motion) }
$$

where the arrows represent a relation of logical entailment. ${ }^{14}$ All Newtonian abduction of the type of primary interest here is of this particular form, involving a framework, a concrete model, and generic equations of motion. It derives something (the theory) that is in a sense "logically intermediate" between the other two terms in the formula, abstract framework and concrete models, intermediate in the sense that one standardly thinks of the framework as "implying" the generic equations of motion, and then the generic equations of motion as implying the individual models, which one then identifies with the concrete models. I will from hereon sometimes speak simply of 'abduction' or 'abductive reasoning' and 'abductive propositions'; unless explicitly stated otherwise, it should be understood that I mean Newtonian abduction. ${ }^{15}$

Contrast this with induction, IBE and deduction. A common form of induction is: the construction of specific equations of motion as a kind of generalization based on many concrete models. A common form of IBE is: the postulation of a set of specific equations of motion based on argument to the effect that the equations of motion provide a good explanation of the concrete models. A common form of deduction is: the derivation of an individual model from specific equations of motion, auxiliary hypotheses, and initial conditions. Its logical form is

(specific equations of motion) \& (initial conditions) \& (auxiliary hypotheses) $\Rightarrow$ individual model

Indeed, this typifies what in philosophy standardly goes by the name of hypothetico-deductive (HD) reasoning. I will often use 'HD' as shorthand for all kinds of deductive reasoning and other forms of inference (such as statistical) more or less conforming to this schema. ${ }^{16}$ One of the most

\footnotetext{
${ }^{14}$ The abductive proposition (3.1) has the logical form of what Carnap (1936, pp. 441ff.) called a conditional definition. I discuss the import of this below in $\S 4$.

${ }^{15}$ There are other types of Newtonian abduction, involving for example the derivation of specific equations of motion from generic equations of motion and concrete models. One can, for instance, determine the ratio of Hooke's constant to the mass of a spring from a concrete model in conjunction with the generic equations of motion for a simple harmonic oscillator. This is a simple-minded example of what Harper (2011) and Smith (2014) call theorymediated measurement. More substantive examples are the determination of the relative masses of the planets in Newton's derivation of universal gravity and the determination of $\omega$ in Brans-Dicke theory by Shapiro time-delay (Harper 2011). In these cases, the logical form of the reasoning is the same as in Newtonian abduction. The difference is in the contents of the terms in the formula: the antecedent is the theory (e.g., Newtonian gravitational theory), not the framework; the lefthand side of the biconditional is still the concrete model; but the righthand side are the values of the parameters being determined. I discuss this further in $\S 4$. One can also consider a weaker form of Newtonian abduction: not a biconditional with a single theory (a single set of generic equations of motion), but rather a biconditional with a family of related theories. Later in this section, we will see an example of this in the discussion of Newton's framework for the investigation of light and color.

${ }^{16}$ I do not claim that all non-abductive forms of deductive reasoning and other similar forms of inference in science are HD; because details of difference in their form do not matter for my purposes, I ignore them.
} 
important differences between this sort of HD reasoning and Newtonian abduction is that the concrete model plays no role in the HD reasoning itself. It rather comes into play only after the reasoning is complete, in the attempt to identify the deduced individual model with a concrete model in order to test the prediction. The reason for this is simple: a theory, in conjunction with initial data, can at most entail an individual model; it cannot entail structured data derived from an actual experiment. In Newtonian abduction, to the contrary, the identification of a concrete model with an individual model forms an essential part of the reasoning itself that results in the theory. This is possible because one has already the concrete model in hand, as one does not in HD. I discuss the consequences of this in more detail in $\S 4$. An example will help illustrate the differences among the forms of reasoning, as well as illuminating perhaps the most important feature of Newtonian abduction, viz., that the derivation is a logical entailment of a particular form, as with HD but not induction nor IBE.

In one of the most important steps in his argument in Principia for universal gravitation, Newton showed in Book III that the magnitude of the force acting on the planets in their orbits about the sun must be proportional to the squared inverse of the distance of the planet from the sun, and that the direction along which the force acts must lie along the line connecting the center of each planet and the sun, pointing towards the sun. To do this, he first proved in the abstract framework of the system of dynamics he had developed, as consequences of his Laws of Motion, that a system of bodies in circular orbits about the same central body, whose orbits manifest a certain set of fixed relations both individually and among themselves (Kepler's Area and Harmonic Laws), must all experience a force obeying an inverse-square law directed towards the central body. ${ }^{17}$ This was a purely mathematical proposition, forming part of the abstract structure of the framework. He then noted, based on the concrete models of the planetary orbits constructed $\grave{a}$ la Kepler from the best available astronomical observations of the day, that the essentially circular orbits of the planets about the sun manifested both individually and among themselves the relevant relations. He concluded that they experienced a force directed towards the sun, obeying an inverse-square law. ${ }^{18}$ In other words, using the resources of his abstract framework he abducted the generic form of the system's equations of motion from their known concrete dynamical evolutions. ${ }^{19}$

In the derivation, Newton did not use Proposition I, Book I, which says that a centripetal force implies the Area Law, to conclude that the force on the planets was always directed along the line

\footnotetext{
${ }^{17}$ The Area Law states that a planet in its orbit around the sun sweeps out equal areas in equal times, the area swept out being that of the region through which the line from the planet to the sun moves. (In modern terms, this is equivalent to the conservation of angular momentum.) The Harmonic Law states that, given the elliptical form of the orbits, the ratio of the square of the orbital period to the cube of the semi-major axis is the same for all planets.

${ }^{18}$ The analysis of his arguments up to this step captures the heart of what is known in the literature as Newton's "deduction from the phenomena" (Harper 1990; Worrall 2000). It is in this sense that the standard account of deduction from the phenomena captures part of Newtonian abduction. As subsequent discussion will make clear, however, the standard account does not capture the full logical structure of Newtonian abduction, and thus cannot support the confirmatory weight I attribute to it.

${ }^{19}$ There are several subtleties of the derivation I gloss over, such as his use of Corollaries VI and VII to Proposition IV in Book I, which are propositions for concentric circles, not ellipses as he knew the planetary orbits to be. The use of those propositions was justified because, when a planet in an elliptical orbit is at a distance equal to the semi-major axis (i.e., when it is 90 or 180 degrees from aphelion), then its centripetal acceleration exactly equals that of a planet in a uniform motion circular orbit of that radius and having the same period, and that suffices for the use of the corollaries. See Stein (1990) and Stein (1994, pp. 639ff.) for more detailed discussion of the logical structure of Newton's reasoning, and exposition and explanation of those sorts of subtleties.
} 
joining the center of the planet to that of the sun; that would have been classic HD reasoning. He rather uses Proposition II, Book I, which says that the Area Law implies a centripetal force of the right kind. He then invokes the fact that the concrete planetary models satisfy Kepler's Harmonic Law to deduce that the gravitational force between celestial bodies varies as the inverse of the square of the distance between them, based on Corollary VI of Proposition IV, Book I. Similarly, he does not use the converse, that an inverse-square force implies the Harmonic Law, which is a theorem as well (proven in the same corollary), to argue for the inverse-square form of the force. Again, that would have been HD reasoning. Finally, he did not calculate, for each planet individually, that an inverse-square law would produce the observed orbit, and then conclude based on these instances that all relevantly similar bodies would also experience such a force. That would have been induction.

That the planets obey the Area and Harmonic Laws are propositions derived from the Keplerian structuring of the direct astronomical observation of the planets' positions over time; that fact, in conjunction with the Newtonian framework (the Laws of Motion), implies part of the form of the generic equations of motion (the force), that it be centripetal, directed towards the Sun, and vary as the inverse-square of the distance. The argument that the force is proportional to the product of the mass of each planet and the sun follows similar reasoning. Newton made use of Proposition I, Book I, that the centripetal form of the force implies the Area Law, and that part of Corollary VI to Proposition IV, Book I, showing that the inverse-square form of the force implies the Harmonic Law, only after the derivation of universal gravity was complete, starting at Proposition XIII, Book III, with what he referred to as an "a priori" deduction of the motions of the celestial bodies starting from the "principles" of his theory, i.e., the generic equations of motion derived in the abductive argument. This explicitly shows that the logical form of the relations among the framework, the concrete model, and the generic equations of motion is that of Newtonian abduction: a conditional with the framework as the antecedent, and a biconditional between the concrete model and the generic equations of motion as the consequent, conforming to formula (3.1). This is not ampliative reasoning in the sense that induction or IBE is. It is logical entailment. ${ }^{20}$

Newtonian abduction is thus the explicit integration of framework and concrete experiment in such a way as to produce theories guaranteed to be appropriate and adequate in all ways, including predictive accuracy, for treating the phenomena used as the basis of the abduction, at least up to the margin of error of the concrete models. ${ }^{21}$ In order to extend the scope of the theory to show its propriety and adequacy for other physical systems one has reason to think are of the same genus, one must perform further reasoning, either of the abductive or HD type. This is exactly what Newton did in the remainder of Book III, using abductive reasoning to show that comets and other

\footnotetext{
${ }^{20}$ It is important to note that, strictly speaking, Newton's initial "deduction" does not work, because, as Newton well knew, the concrete models he relied on did not exactly, only approximately, instantiate the Keplerian relations. Here is where what Harper (2011) calls Newton's method of successive approximations comes into play. In effect, Newton decided to assert the validity of the simple abductive proposition and then push it as far as he could by successive refinements of the approximative models, each one markedly improving on the previous. (See Stein 1967, pp. 177-180 for a concise and lucid discussion of Newton's guiding methodological principle here and the way he applied it.) It is worth remarking as well that HD cannot accommodate this method, as it can do nothing but strike the hypothesis from the record of viable candidates at the first glimpse of such inconsistency with the data.

${ }^{21}$ It would be an interesting project to characterize the necessary and sufficient conditions on the structures (topological, algebraic, geometric, etc.) a framework imposes on its family of theories required for the framework to support Newtonian abduction.
} 
celestial bodies also obeyed the generic equations of motion of Newtonian gravitational theory (following from the approximately parabolic form of their orbits), and the same for terrestrial systems as well (free fall, the motion of pendulums, and so on).

It should be clear that Newtonian abduction works as logical entailment only because I have put into the framework from the start all the practices and principles that allow one to meaningfully bring theory and experiment into contact with each other so that the former may be used to interpret the latter, and the latter may be used to constrain the former, i.e., so that data may be structured in such a way as to be directly comparable with, and even identified with, solutions to the equations of motion (i.e., the identification of concrete with individual models). This will include, inter alia:

- mathematical structure, relations and formulæ in addition to the abstract equations of motion (e.g., in Newtonian mechanics that $\vec{v}={ }_{\mathrm{df}} \dot{\vec{x}}$, that mass is additive, that spacetime has a flat affine structure, and so on);

- standards of good argumentation for different genera of physical systems (accepted approximative techniques for solving equations and constructing simplified individual models, sound heuristics for informal arguments, and so on);

- families of accepted experimental and observational practices for systems of different genera;

- rules for connecting experimental outcomes with formal propositions (semantics, pragmatics, principles of representation, construction of concrete models from raw observations, rules for interpreting structured data using the conceptual vocabulary of the framework, rules for reckoning expected experimental precision and error, and so on);

- rules of evidential warrant (what can be evidence, how to apply it, reckoning of error tolerance, and so on);

- and guidelines for judging the legitimacy of proposed modifications, extensions, and restrictions of all these.

In other words, the framework includes all physical and methodological principles and practices required to bring theory and experiment (in the form of structured data) into substantive, fruitful contact. ${ }^{22}$ Otherwise the entailment of the biconditional would not be valid. Most of this will be difficult if not impossible to articulate and record in an exhaustive and precise way, so as to lend itself to use in formal philosophical investigations. We must trust that all such collateral principles and practices are there, and can be, now and again, each more or less precisely articulated as the occasion demands. The same holds true, however, for formal reconstructions of all forms of reasoning in science (e.g., the 'auxiliary hypotheses' of $\mathrm{HD}$, which always hide an ugly mob of philosophical sins).

Does all this make the idea of a framework too vague? We simply throw in everything that will "make the abduction work"? Is it, e.g., a methodological principle of Newtonian mechanics that

\footnotetext{
${ }^{22}$ On this view, the entirety of a framework is a dynamic entity, as are individual theories, evolving over time as new theoretical and experimental techniques and practices are developed and accepted, and so theories themselves will be as well. I think this is the right way to think about these matters for many if not most purposes in those parts of philosophy of science studying scientific theories. The contemporary practice of treating theories as static, fixed entities, especially in work of a more technical and formal character, can lead to serious philosophical error. An adequate semantics of a theory, for instance, should reflect and accommodate its dynamic nature.
} 
one prefer a single law (inverse-square) for all the planets, rather than, perhaps, $\frac{1}{r^{2.0000001612}}$ for Mercury, the figure one arrives at by using the Precession Theorem (explained below) on the most accurate data we have for Mercury's orbit? ${ }^{23}$ To do the same to account for the Moon's motion around the Earth, again based on the Precession Theorem, would yield a force proportional to $\frac{1}{r^{2+\epsilon}}$, where $\epsilon<0.00000004$, clearly inconsistent with the exponent required for Mercury, as shown by the analysis of Brown (1904), of the Hill-Brown lunar theory. (See Wilson 2004 for discussion.) And one would then go on to choose a slightly different force law for the attraction of each individual planet to the sun, and for the attraction of each comet to the sun, and so on. All such laws could be derived by abductive reasoning in the framework of Newtonian mechanics. Choosing those multiple force laws, of course, would eo ipso be to treat Newtonian gravitational theory as disconfirmed. But there are methodological principles in the Newtonian framework, explicitly in this case, that tell us to prefer the single law for all planets, and indeed for all gravitating bodies, viz., Newton's Rules of Reasoning in Natural Philosophy (Newton 1726, Book III), specifically Rules II and IV, which he invokes in the argument in Book III for universal gravitation:

Rule II "[T]o the same natural effects we must, as far as possible, assign the same causes."

Rule IV "In experimental philosophy we are to look upon propositions inferred by general induction from phenomena as accurately or very nearly true, notwithstanding any contrary hypotheses that may be imagined, till such time as other phenomena occur, by which they may either be made more accurate, or liable to exceptions."

The argument that these Rules imply, or at least militate in favor of, a preference for a single force law is subtle and involved, and beyond the scope of this paper, but I hope it to be at least intuitively clear that they do so. ${ }^{24}$

The Precession Theorem (Principia, Book I, Proposition XLv, and its corollaries), and the use Newton and later investigators put it to, display the great power of Newtonian abduction as a form of reasoning, allowing one to draw conclusions much stronger and more robust in several ways than any HD, inductive or IBE reasoning can support. The Precession Theorem, in essence, says that, if a body is in nearly circular orbit around a central body, and its perihelion precesses, then the rate of precession measures the difference between an inverse-square force law and the force law governing the orbit. If such an orbit has no precession-i.e., if it is closed-then it obeys an exact inverse-square law. If an orbit has small precession, then the force law will be of the form $\frac{1}{r^{2+\epsilon}}$, for some small $\epsilon$ proportional to the amount of precession. The converses hold as well: if bodies do not obey an exact inverse-square force law, then there will be precession, and its rate will measure the deviation from inverse-square. All the planetary orbits are, in the precise sense Newton defines, nearly circular and exhibit small precession. Based on the best observational data, Newton knew that the orbit of no planet is exactly a closed curve. By calculating the expected perturbative gravitational influences of the other planets (primarily Jupiter) on the orbit of each planet, he

\footnotetext{
${ }^{23}$ See Hall (1894) for the initial proposal of this, and, e.g., Newcomb (1895a, 1911) for further championing of the idea.

${ }^{24}$ That Newton uses the term 'general induction' in Rule Iv has no bearing on my arguments. The word 'abduction' did not exist then, and, in any event, as is made clear by Newton's gloss on the rule following its statement, Newton is using 'general induction' to refer, among other things, to the pattern of reasoning he employs in deriving his theory of universal gravity, which I claim is in fact Newtonian abduction.
} 
showed that the precessions would be negligible (on the order of arcminutes per century), and so abductively concluded from the Precession Theorem that this provided further evidence for the inverse-square law (Book III, Propositions XIII and XIV). ${ }^{25}$ The only exceptions to this are the perturbative effect Jupiter and Saturn have on each other when they are close to conjunction, and the irregularities in the moon's motion (e.g., librations arising from precession) caused by the non-negligible combined effects on it of the oceans' tides and the Sun. Again, he was able to show by abductive argument based on the Precession Theorem that more finely detailed concrete models of the orbits, including the irregularities from perturbations, yield the inverse-square force law (Book III, Propositions XIII, XVII, and XXII).

Le Verrier (1845) subsequently improved on Newton's reasoning. Using more accurate data and more detailed perturbative models of the effects of other planets' gravitation on a given planet's orbit, he showed that subtracting the effective perturbative forces would yield closed orbits for all the planets (within the bounds of error for the available data), thus giving even stronger confirmation of the inverse-square law by abduction based on the Precession Theorem. ${ }^{26}$ This is a powerful improvement on Newton's initial argument I sketched above for the form of the gravitational force law. One constructs the initial individual models used in deriving the inversesquare form, closed nearly circular orbits, by ignoring known features of the concrete models that deviate from the individual models, viz., their precession. One then constructs new individual models that take into account more of the known features of the concrete models, and subsequently shows by abductive reasoning that those yet more accurate individual models yield the inversesquare law, when corrective perturbations are accounted for.

The form of the argument was not:

1. if there are deviations from inverse-square, then there will be orbital precession (individual models);

2. there is observed orbital precession (concrete models);

3. therefore, I will find the right force law from which I can deduce the observed precession (individual models);

\footnotetext{
${ }^{25}$ More precisely, he used Proposition LXVI and its corollaries (Book I), which are themselves derived from the Precession Theorem. The content of the Precession Theorem itself carries the burden of the argument.

${ }^{26} \mathrm{In}$ fact, this is not true for Mercury, as Le Verrier well knew. There was an extra 39 arcseconds $\left(39^{\prime \prime}\right)$ per century of precession that his calculations could not account for. He labored for the next 14 years to produce a mechanism to explain the discrepancy, even postulating hitherto unobserved celestial bodies and other such ad hoc devices, but nothing worked (Le Verrier 1859). Indeed, by the end of the 19th Century the inexplicability of the aberrant precession was such a great embarrassment that many eminent physicists had already concluded that Newtonian gravitational theory could not be fundamentally correct, even before the development of special relativity (a historical fact that seems to be not so well known as it ought), based entirely on abductive use of the Precession Theorem. See Newcomb (1895b), Newcomb (1895a), Newcomb (1905) for an extended discussion and summation of the experimental knowledge of the aberrant precession at that time, when the anomalous amount of Mercury's precession was finally fixed at $43^{\prime \prime}$ per century, and see Freundlich (1915) for an exhaustive argument that Newtonian gravitational theory could not account for it. To get a sense of how small the angle $43^{\prime \prime}$ is, imagine the appearance of the diameter of a penny from a distance of about 30 miles. The apparent length of its diameter on the eye, projected back to the penny, subtends an angle of that size. It is a testament to the profound confirmatory entrenchment of Newtonian gravitational theory in particular at the time, and Newtonian mechanics in general, that a discrepancy of this infinitesimal angle per century in a planetary orbit caused such consternation in and provoked such labor from the leading lights of the scientific community for more than 70 years. Of course, we now know that the error arises from general relativistic effects, and cannot be accommodated by Newtonian gravitational theory.
} 
4. I will do so by deducing all the expected perturbative effects of all other planets on each planet's orbit for different force laws individually (individual models);

5. I will thus find the law that implies that those perturbative effects give rise to the observed precession.

That would be HD reasoning. Rather, the argument runs thus:

1. there is observed orbital precession (concrete models);

2. it is a mathematical theorem in the framework that there is orbital precession if and only if there is deviation from inverse-square, and the amount of precession determines the amount of deviation (abstract structure);

3. I will construct individual models that include the perturbative effects from the other planets, based on the observed orbits (the concrete models);

4. it then follows abductively, from the concrete models, the abstract equations of motion and the Precession Theorem, that there is a unique force law which is such that, for all planets, when the perturbations calculated from the force law are subtracted from each planet, and that planet is modeled as a two-body system orbiting around the sun, then the orbit of the two-body problem determined ab initio by the force law is the same as the orbit determined by subtracting all the calculated perturbations, and that this holds if and only if the concrete model is uniquely identifiable with an appropriate individual model;

5. the resulting orbits for all planets are closed, i.e., there is no precession;

6. by abduction, this holds if and only if the unique force law is inverse-square.

It is clear from the reconstruction, moreover, that abductive reasoning supports reasoning based on subjunctive conditionals. It allows one to deduce conditionals of the form "if the phenomena were different in this particular way, the laws would be thus and so". In fact, it allows one to deduce subjunctive biconditionals: "the phenomena would be different in this particular way if and only if the laws were thus and so". It thus allows one to set principled bounds on how far wrong the abductively derived laws can appear to be, and still be within the known margins of error of extant observations. Even more, it shows that construction of more finely detailed, more accurate concrete models also yield the same force law by directly showing, in one fell logical swoop, that no other force law could accurately represent the finer, more accurate models, within the margin of error believed at the time. ${ }^{27}$

Subjunctive reasoning of this kind provides extraordinarily strong epistemic warrant for the abductively derived laws, as it shows how far the concrete models constructed from detailed observations can deviate from simpler individual models while still remaining within the regime of

\footnotetext{
${ }^{27}$ Indeed, Newton had even more instances of such reasoning than only that based on the Precession Theorem. According to Corollary vis to Proposition IV, Book I, the Harmonic Law also provides support for the derivation of such subjunctive conditionals. Over and above the biconditional between the Harmonic Law and the inverse-square form of the force law, the corollary shows that the periods of the planetary orbits are proportional to a power greater than the Harmonic Law's 3/2 power of the semi-major axes if and only if the centripetal forces fall off more rapidly than the inverse squared law, and contrarily that the periods are proportional to a power less than $3 / 2$ of the semi-major axes if and only if the centripetal forces fall off more slowly than the inverse-square law. See Harper (1999) and Harper (2011, pp. 114-120) for discussion.
} 
applicability of the laws (Harper 2011; Smith 2014). This is not possible in the HD scheme. In that form of reasoning, one can show the validity only of conditionals of the following form: "if the laws were different in this particular way, the phenomena would be thus and so". But that shows nothing of epistemic import. One cannot use such conditionals to set bounds on how wrong simple individual models can be and still fall within the regime of applicability of the proposed laws when compared to more detailed concrete models. Indeed, one cannot use such conditionals to derive any substantive proposition at all about the actual world. This is a striking example of the power of abduction as compared to standard HD. ${ }^{28}$

Newton's (1672) framework for theories of light and color makes a fascinating case study for teasing out the many subtleties, layers and facets of these ideas. ${ }^{29}$ That framework allowed for the articulation of many different kinds of theories, both wave and particle, and facilitated the switch from the dominant particle theory to a new wave theory at the beginning of the 19th Century, at the hands of Young and Fresnel. This framework, however, is abductive in a subtler way than the "standard form" I have sketched, at least in its actual historical applications. There was no biconditional between the concrete models and either particle or wave theories up until, arguably, the 19th Century, when diffraction decisively favored wave theories. Even after the work of Young and Fresnel, when the framework was pared down to accommodate only wave theories, the available data did not allow the abduction of a single, fully determinate theory until Maxwell's electromagnetic theory of light was confirmed by Hertz's experiments on electromagnetic radiation. The derivation by Maxwell (1864) of his full electromagnetic theory, abductive to its core, required a framework far more comprehensive than Newton's for light and color, though it did incorporate Newton's framework as a sub-framework, so to speak. ${ }^{30}$ Newton's framework for light

\footnotetext{
${ }^{28} \mathrm{~A}$ good example of this from contemporary physics is the parametrized post-Einsteinian framework of Yunes and Pretorius (2009), which manifestly supports subjunctive reasoning of this form, with the explicit intent of sharpening the recent observation of gravitational waves by LIGO (Abbott, B. et al. (LIGO Scientific Collaboration and Virgo Collaboration) 2016) as tests of general relativity, by providing a framework within which one can probe for deviations from general relativity's predictions in a parametrized, controllable form: the dynamics of the observed coalescence of the binary black hole system deviates from general relativity's models in this way, by this parametrized amount, if and only if the observed gravitational waveform exhibits this quantifiable feature.

${ }^{29}$ For an exposition of Newton's framework for light and color, and the investigations and abductive reasoning that led him to it, see Curiel (2001) (though I do not refer to the form of reasoning as Newtonian abduction in that paper), and for a more extensive and deeper discussion of those investigations, with a direct bearing on the relevant issues, see Stein (unpublished-a), Stein (unpublished-b).

${ }^{30}$ Due to limitations of space, I cannot give a detailed argument that Maxwell's reasoning for his equations governing the electromagnetic field is abductive. I will note here only that, in Maxwell (1856), his derivation of the equations, including the necessity of the novel term representing the so-called displacement current, at bottom takes the form of a biconditional between, on the one hand, the electromagnetic phenomena observed and regimented by, inter alia, Ersted, Ampère, and (most of all) Faraday, and, on the other hand, what we now call Maxwell's equations, where the biconditional is implied by a Newtonian framework comprising the theory of a particular kind of Newtonian fluid. His final, complete derivation in Maxwell (1864) also has this logical form, though the biconditional's antecedent is a Newtonian framework comprising a completely abstract theoretical representation of a medium whose dynamical behavior is governed by a Newtonian form of elasticity. In Maxwell (1891), he abductively derives the equations in the framework of Lagrangian mechanics. I emphasize that these claims are crude and naive in the extreme, requiring detailed historical and technical exegesis for their complete elucidation and defense. Nonetheless, I also claim that they capture the heart of the matter. In the same vein, it is worth considering the argument of Hertz (1884) concerning Helmholtz's formulation of electromagnetism that he intended to be neutral between Maxwell's theory and Weber's action-at-a-distance theory (in other words, Helmholtz's formulation of a framework subsuming both). Hertz argued, in effect abductively, that Helmholtz's framework is in fact inconsistent
} 
and color, therefore, in itself is perhaps best described in its historical applications as supporting not the abduction of theory from framework, but rather the abduction of pared-down frameworks from a more general framework. This all raises fascinating and deep questions about the possible relations among different frameworks, questions that I think are interestingly different from similar ones about inter-theory relations, but which are in any event beyond the scope of this paper.

I may have given the impression so far that abduction is a form of reasoning peculiar to Newtonian mechanics (on the assumption that Maxwell's abduction of his theory of electromagnetism was formulated in a Newtonian framework, as I claim). To the contrary, the history of physics is replete with examples of abduction, and it is still used today far and wide in all branches of physics. Indeed, I do not think it is an exaggeration to say that the large majority of revolutionary advances in physics were based on abductive reasoning. I do not have the space here to make that case fully, so I will content myself with listing exemplary instances across many branches of physics.

1. In Lagrangian mechanics, one abducts the generic equations of motion by applying a variational principle (encoding the Euler-Lagrange equation, the abstract equations of motion) over the family of known individual models on the generic space of states. (I discuss this example in more detail in $\S 4$.)

2. In Hamiltonian mechanics, one derives the form of the Hamiltonian from the abstract form of Hamilton's equation in conjunction with the form of the phase portraits of the systems' evolutions in phase space (the individual models). A trivial example: construct the phase portrait of a simple harmonic oscillator by continually measuring its position and momentum; the resultant set of points forms a circle in phase space (ignoring constant coefficients); it follows then directly from the abstract form of Hamilton's equation that the Hamiltonian must be $p^{2}+q^{2}$, and by plugging this into the abstract form of Hamilton's equation one has abducted the generic equations of motion.

3. In quantum mechanics, one abducts the form of the Hamiltonian from the abstract Schrödinger equation in conjunction with the paths of unitary evolution on Hilbert space (the individual models); the procedure is almost identical to that in Hamiltonian mechanics. This is how the Hamiltonian for the Hydrogen atom, e.g., is derived.

4. In quantum field theory, one abducts the form of the Lagrangian from observed symmetries, playing in this case the role of the concrete models (Sakurai 1964).

5. In Boltzmannian statistical mechanics, one abducts the form of the Maxwell-Boltzmann distribution from the abstract equations of motion for statistical quantities, in conjunction with constraints imposed by empirically observed properties of the phenomena of thermodynamical equilibrium (concrete models) (Sommerfeld 1964). The same holds for derivations of the microcanonical, canonical and grand canonical distributions in Gibbsian and quantum statistical mechanics (Fowler and Guggenheim 1949; Landau and Lifschitz 1980). (Malament and Zabell 1980 provide a philosophically compelling explanation of the way that the physical

with Maxwell's theory, in so far as it cannot represent the existence of free electromagnetic radiation, which itself abductively favors Maxwell's theory. Again, a detailed defense of this claim is beyond the scope of this paper. 
characteristics of Gibbisan equilibrium for isolated systems are necessary and sufficient for the microcanonical distribution for appropriate physical systems; Sorkin 1979 provides great physical insight into the way that appropriate further characterizations of equilibrium provide necessary and sufficient conditions for the canonical and grand canonical distributions respectively.)

6. In ordinary thermodynamics, one abducts the principle of entropy non-decrease from the Kelvin Postulate (the abstract "equation of motion") in conjunction with the existence of irreversible processes, viz., the concrete models (Fermi 1937). This example shows that "equation of motion" can be broadly construed in the context of abduction, as I remarked in footnote 3 .

7. In general relativity, one abducts the FLRW cosmological models and the Schwarzschild solution from the Einstein Field Equation and the observed (or postulated) symmetries of the dynamical evolutions of those genera of systems (Wald 1984). ${ }^{31}$

8. In cosmology, the inferences to dark energy and to dark matter, and so the construction of the entire standard $\Lambda \mathrm{CDM}$ ("cosmological constant plus cold dark matter") model of contemporary cosmology, are abductive, starting from the framework of general relativity in conjunction with the concrete models constructed from observations such as the velocitydispersion relations of galaxies, the isotropy of the cosmic microwave background radiation, and the large-scale accelerating expansion of the universe (Weinberg 2008).

In all of these cases, the abductive form of the entailment also supports reasoning based on subjunctive conditionals, as in the case of Newtonian gravitational theory. This is almost trivially easy to see in the cases of Lagrangian mechanics, Hamiltonian mechanics, quantum mechanics, and quantum field theory. In thermodynamics, it is easy to show based on the abduction that, if there were no irreversible processes, then the principle of entropy non-decrease would hold only trivially (i.e., entropy would never increase). For the FLRW cosmological models of general relativity, deviations from perfect symmetry (anisotropies, inhomogeneities) in the concrete models subjunctively yield different spacetime models, e.g., Bianchi-type models (Griffiths and Podolský 2009, ch. 22) or Szekeres models (Szekeres 1975), depending on the form of the deviations from symmetry. In the $\Lambda \mathrm{CDM}$ model of cosmology, the percentages of total mass-energy constituted by dark energy and dark matter are subjunctively related to the values observed for velocitydispersion, accelerating expansion, and other such physical quantities measured and represented in the concrete models of the universe (Weinberg 2008; Frieman, Turner, and Huterer 2008). The only case in which it is not clear to me that this is so is Boltzmannian statistical mechanics. Distributions for deviations from equilibrium can be derived, but I do not know whether the form of reasoning in that case supports the picking out of unique distributions for given deviations from equilibrium. I suspect it does not. It would be of interest to figure this out.

\footnotetext{
${ }^{31}$ It is a little delicate to explain the way in which a family of spacetime models such as the FLRW or Schwarzschild ones is relevantly like a set of generic equations of motion, but one can do this, and when one reconstructs the reasoning involved, say, in proving Birkhoff's Theorem (which implies the uniqueness of Schwarzschild spacetime given the assumed symmetries), it is indeed abductive in form. If you object to the claim that general relativity is a framework, then think of this as the abduction of specific equations of motion from concrete models and generic equations of motion (general relativity considered as a theory, not a framework).
} 
Now that the idea has been explicated and discussed, I conclude the section by giving the promised sketch of an explanation of the way that Newtonian abduction is similar enough in form and intent to Peirce's original conception of abduction to warrant using the same name for it. As spelled out in Peirce (1878a), Peirce (1903), Peirce (1955), the basic idea of abduction (or 'hypothesis' as he sometimes calls it) is that, from the assumption of a general rule $F$ and a basic state of affairs $C$ relevantly related to the rule, a proposition $T$ is derived by abduction in his sense if it is such that the conjunction of $F$ and $T$ logically entails $C$, or else that $T$, on the assumption of $F$, makes it probable that or offers a possible explanation of $C$. (Again, Peirce places no requirement of "goodness" on $T$ for it to be produced by abduction.) If, in my terms, $F$ is the framework (general rule), $C$ the concrete model (basic state of affairs), and $T$ the theory (proposition), then the similarity in form and intent between Peircean abduction and Newtonian abduction is clear: in Newtonian abduction, the assumption of $F$ and $T$ logically entails $C$, at the same time as guaranteeing that the conjunction $F$ and $C$ entails $T$. One can also view Newtonian abduction as being similar to Peirce's original proposal in so far as one might think that the theory derived by Newtonian abduction gives an explanation of the phenomena that yielded the concrete model, given that the phenomena is treatable by the framework. Newtonian abduction, therefore, differs from Peircean abduction primarily in being logically stronger: the derived proposition in the former is logically entailed by the "premises", which is not in general the case in the latter.

\section{Framework Confirmation}

In order to characterize the kinds of confirmation I claim accrue to frameworks from abduction, it will be useful to begin with a discussion of some observations on the topic by James Clerk Maxwell. He is the only scientist or philosopher I know, besides Newton, who explicitly recognizes Newtonian abduction as a separate form of reasoning in science and articulates its form.

Maxwell (1876, p. 309) explains the idea clearly:

[T] he importance of [the] equations [of motion] does not depend on their being useful in solving problems in dynamics[, i.e., in deducing predictions of future behavior when the forces acting on a system are known]. A higher function which they must discharge is that of presenting to the mind in the clearest and most general form the fundamental principles of dynamical reasoning.

In forming dynamical theories of the physical sciences, it has been a too frequent practice to invent a particular dynamical hypothesis and then by means of the equations of motion to deduce certain results. The agreement of results with real phenomena has been supposed to furnish a certain amount of evidence in favour of the hypothesis.

The true method of physical reasoning is to begin with the phenomena and to deduce the forces from them by a direct application of the equations of motion.

It may seem that Maxwell contradicts himself here. If we already know the equations of motionas we must, if we are to "directly apply" them to deduce forces from phenomena - then surely we know already the forces as well, since the equations of motion are formulated in terms of the forces, and only thence one deduces the phenomena, à $l a \mathrm{HD}$. The context of the passage, however, makes 
clear that Maxwell has a conception of "equations of motion" in mind different than that involved in employing a representation of known forces to deduce resultant motions using generic or specific equations of motion in conjunction with initial data. What he calls here the "equations of motion" are what I call, in essence, the abstract equations of motion of a framework, and "the fundamental principles of dynamical reasoning" are, in effect, the abstract structures of the framework itself, as encoded in the abstract equations of motion and other mathematical relations and principles, for example, that mass is additive in Newtonian mechanics.

Maxwell began the paper (more of a brief note than a paper, really, only two pages in length) by adverting to the method Lagrange introduced in the late 18th Century in his Mécanique Analytique, the essence of what we now know as Lagrangian Mechanics, and contrasting it to that involved in the deduction of dynamical behavior from (in my parlance) generic or specific equations of motion in conjunction with initial data. Recall that the Euler-Lagrange Equation is the heart of Lagrangian Mechanics (Curiel 2014). This equation results from the demand that the dynamically possible evolutions manifested by a physical system optimize, according to the principles of the calculus of variations, a certain integral function of the dynamic quantities, the action. Thus, the Euler-Lagrange equation is, in my sense, the abstract equation of motion in the framework. To know the individual models, then, of a genus of physical system (derived, say, by identification with concrete models) allows one to abduct the generic equations of motion as a particular instantiation of the Euler-Lagrange equation: the variational principle entails that the individual models are a particular family of paths on the space of states if and only if the action is of a particular form (and so the generic equations of motion are of a particular form). ${ }^{32}$ Thus, this method of deriving the equations of motion exemplifies Newtonian abduction: knowing the concrete models, one identifies them with dynamically possible paths on the space of states (the individual models), and thence one abducts the form of the forces acting on the system (the generic equations of motion) by applying the machinery of the abstract equation of motion to the paths. Such a derivation does not so much provide evidentiary support for the resulting generic equations of motion as being the most correct among a field of competitors or something of the sort. It rather demonstrates, eo ipso, that the derived equations have the structure appropriate for modeling the concrete dynamical evolutions of the system, and nothing more - because the structure of the derived generic equations of motion is directly determined, biconditionally, by the structure manifest in those dynamical evolutions (the concrete models, identified with the individual models).

Newtonian abduction, therefore, shows, or captures, or elucidates the mathematical structure manifest in the phenomena. That part of the world treated by the abducted theory, as investigated by appropriate experimental techniques, directly evinces the abstract structure the framework exhibits and requires. ${ }^{33}$ A good example of this kind of structure is given by the space of states and the dynamically possible evolutions of an abstract Newtonian system, one treated by a theory

\footnotetext{
${ }^{32}$ For a graphic illustration of the method, see Curiel $(2014, \S 4)$, in particular the discussion of how one can read the generalized forces off from the form of the second-order vector fields on the tangent bundle of the configuration space for a given genus of physical system, those second-order vector fields representing the allowed dynamical evolutions of the system, i.e., its individual models.

${ }^{33}$ One can therefore understand Newtonian abduction as providing grounds for the position of the "sophisticated instrumentalist" as characterized by Stein (1989). (I thank Tom Pashby for pointing this out to me.) This does not, however, imply that Newtonian abduction by itself militates in favor of instrumentalism, nor realism either for that matter.
} 
whose generic equations of motion are an instantiation of Newton's Second Law (Curiel 2014). The algebraic and differential structure of the Second Law itself imposes the structure of a vector space on the family of all vector fields on the abstract space of states representing possible interactions ("imposed forces"). The family of vector fields representing dynamically possible evolutions of the system itself then accrues the structure of an affine space modeled on that vector space. It then follows that the abstract space of states is naturally isomorphic to the tangent bundle over the system's configuration space (i.e., there is an isomorphism distinguished by the physics, in this case by the existence of a privileged dynamical vector field representing "free evolution"). Any theory abducted in the Newtonian framework inherits these structures. In particular, when one compares different concrete models of the same system, say, ones constructed at different times when it is experiencing forces of different magnitudes, one will find the appropriate affine and additive structures on that family of concrete models (when the concrete models are appropriately interpreted using the conceptual machinery of the framework). ${ }^{34}$ This is easy to verify in, e.g., Newtonian gravitational theory. Stein (1994, p. 639, emphases his) makes the point in a trenchant way, explaining in illuminating detail how Newtonian gravitational theory is "a theory of a mathematical structure discernible in the world of phenomena, of observations, of experience."

What Stein says of Newtonian gravitational theory is straightforwardly translated so as to apply to the entire Newtonian framework itself (and indeed to all frameworks) - they provide mathematical structures discernible in the world of phenomena, of observations, and of experience, in such a way as to allow us to exploit those structures for the theoretical representation of physical systems so as to support substantive scientific reasoning of all forms about them. To perform empirically successful scientific reasoning by the direct and ineliminable application of a theoretical structure, however, is to endow that structure with some measure of confirmatory support, by any reasonable standard.

I call this type of confirmation structural confirmation. ${ }^{35}$ It accrues to a framework when one demonstrates that the structures intrinsic to the framework are appropriate and adequate for representing and reasoning about the genera of physical systems the framework purports to treat. It is appropriate if the framework's structures allow one to identify in the relevant sense individual models of a theory in the framework with concrete models of physical systems in the genus treated by the theory. It is adequate if one can use that identification to engage in substantive, successful scientific reasoning about those physical systems, and, moreover, one has good reason to believe that such identifications can be carried out for a much broader range of relevantly similar systems than the ones already treated. Thus, when I follow Stein in speaking of "a mathematical structure [of a framework] discernible in the world of phenomena," I mean that the framework facilitates the successful identification of individual models with concrete models in such a way as to make it possible for its theories to be used in successful reasoning. In particular, I make no claims that structures in the individual models and "in the world itself" are "isomorphic" or "homomorphic" or "similar" in any way above and beyond the fact that the individual models are relevantly identifiable

\footnotetext{
${ }^{34}$ It is important to note that no concrete model on its own has any of these structures; only rich enough families of them exhibit the structures as relations among them.

${ }^{35}$ After I finished this manuscript, I discovered that Kuipers (2001, pp. 208-209) uses 'structural confirmation' to refer to confirmation derived from instantiation of another relation, that of partial entailment, i.e., the probabilistic degree to which $A \vee B$ entails $A$. The two should not be conflated.
} 
with the concrete models, in large part because those structures in the individual models do not form part of the concrete models.

Before continuing the main thread of the discussion, I want to pause for a moment to make clear what I am claiming such an identification of individual with concrete models consists of, and in particular why I claim that it neither presupposes nor implies any substantive isomorphism between the two. There are many ways one can legitimately conclude that an individual model in a theory can be identified with a concrete model constructed from experimental results. In my discussion of the example of the simple harmonic oscillator in Newtonian mechanics, on p. 2, in my introduction of the idea of a concrete model, one identifies the individual model with the concrete model by the brute comparison of the values in each that one has some reason to believe represent the same physical quantities. It is true that, in this case, one identifies a geometric circle in the concrete model with one instantiating an individual model in the theory, and so there is isomorphism in this superficial sense - circle to circle-but there is isomorphism of nothing else. In particular, the individual model has far more structure than a continuously parametrized sequence of values of physical quantities (which exhausts the content of the concrete model), structure accruing to it from the theory that makes it an individual model of that theory. In the case of the simple harmonic oscillator, for example, the individual model has the further implicit structure of the flat affine structure of Newtonian spacetime, which defines the derivative operator with respect to which the individual model is a smooth curve. The concrete model need not have that structure. It really is just a continuously parametrized sequence of values of physical quantities. Even if one were to put a flat affine structure into the concrete model by hand, it would be superfluous, as the identification of the concrete model with the individual model does not depend on it. To take the isomorphism between the two continuously parametrized sequences of values of physical quantities - the only isomorphism available - as a philosophically substantive isomorphism would be nothing more than the sheerest naive verificationism.

In consequence, one can agree with the idea of structural confirmation while remaining agnostic about all issues pertaining to realism and anti-realism, as any good confirmatory relation should allow. In particular, structural confirmation has no necessary connection to the idea of structural realism (though I suspect that champions of the program may want to try to avail themselves of it in their arguments).

It may sound strange to say that Lagrangian mechanics itself admits of confirmation, but I claim it does. Structural confirmation does not give us a reason to believe that Lagrangian mechanics is true. I am not even sure what that claim could mean. Structural confirmation rather gives us reason to believe that the structures intrinsic to Lagrangian mechanics are approriate and adequate for representing many genera of physical systems. We come to believe in its fruitfulness as a ground for successful scientific reasoning of all sorts. That, however, is exactly what standard confirmation does for us with regard to ordinary theories. It is only the realist who believes confirmation also gives a reason to think the theory is true; the instrumentalist is not committed to taking that further step. ${ }^{36}$

\footnotetext{
${ }^{36}$ Of course, everything said of structural confirmation of a framework holds as well, mutatis mutandis, for a theory. Theories also are amenable to structural confirmation, in the same way, by abductively showing that the structures intrinsic to the theory are appropriate and adequate for representing and reasoning about the concrete models of the different species of physical system the theory purports to treat.
} 
Abduction shows that a genus of physical systems is governed by the generic equations of motion and other mathematical relations imposed by a theory in the relevant framework if and only if the relevant space of states and family of dynamically possible evolutions, appropriately identified with the family of concrete models, has that structure. In other words, those systems are appropriately and adequately treated by a theory in the framework if and only if their predicted behavior, both in isolation and in response to the kinds of interactions with the environment allowed by the structures of the theory, accords with the constraints imposed by those structures. This is not only a successful prediction of the theory by the framework - it is also the provision of grounds for the possibility of using the theory to make successful predictions, i.e., a necessary precondition for being able to apply the theory in that way, when the theory is generated by abduction. As such, every successful application of the theory in, e.g., making predictions of the HD-type, endows confirmation on the framework as a whole, when the theory is generated by abduction, since abduction made it possible in the first place.

Newtonian abduction is a relation between theory and experiment that standard accounts of confirmation based on HD-type reasoning cannot accommodate. Structural confirmation, nonetheless, as I just intimated, derives in part from the validation of a proposition that itself entails a proposition largely the same in form as used in the standard accounts of confirmation based on HD forms of reasoning: $H$ (the framework) entails some $E$ (evidence, in this case the biconditional between theory and concrete models).

Indeed, with the addition of two extra assumptions - determinate values for the constant coefficients in the generic equations of motion to turn them into specific equations of motion, and initial data-, and replacing the concrete model in the abductive proposition with the individual model derived from the specific equations of motion and the initial data, the abductive proposition even implies a proposition identical to that used in HD, by discharging one of the directions of the biconditional: the framework entails that $H$ (an abducted theory) entails $E$ (evidence in the form of an individual model that can potentially be identified with a concrete model). Nonetheless, as the description of the way that one can use the abductive proposition to derive one as used in HD makes clear, concrete models play no role in the logic of HD itself, as I remarked in §3 just after formula (3.2). HD predicts an individual model. Concrete models come into play only after the logical derivation of the individual model, in the attempt to identify the deduced individual model with a concrete model, to test the HD prediction. This is why Newtonian abduction automatically shows the theory to be appropriate and adequate for treating the phenomena that yielded the concrete model in a way that HD can never do, since the concrete model itself forms an essential part of the reasoning. Since the identification of individual model with concrete model occurs in HD only after the reasoning has concluded (if it is possible at all), there can be no such necessary conclusion about the propriety of the theory in treating the phenomena.

I suspect that one may be able to extend some of the standard accounts of confirmation, based on HD reasoning, to accommodate Newtonian abduction and structural confirmation, but the conceptual obstacles are formidable. There are as well obvious and prima facie difficult technical obstacles that immediately present themselves. For instance, in any account of confirmation that quantifies it using probabilities, such as Bayesianism, one will have to construct a probability space that includes as elements the framework itself, the theories possibly abductable from the framework, the individual models of those theories, and the concrete models that may be appropriate for 
identification with those individual models. That is not all, however. As the discussion of Newton's and Le Verrier's use of the Precession Theorem shows, the probability assignments on the space will have to respect the non-trivial relations among the theories (topological, algebraic, differential, and so on) that the framework endows on its family of theories, those relations that make Newtonian abduction possible in the first place and are integral in its support of reasoning based on subjunctive conditionals (and so integral to what I call modal confirmation, discussed below). These relations go far beyond the standard $\sigma$-algebra required on a space in which one defines a probability measure satisfying the Kolmogorov axioms. ${ }^{37}$ One will have to show, moreover, that the further structures are consistent in the right ways with the probability structure. These are highly nontrivial problems. ${ }^{38}$

In sum, abduction is a form of reasoning that indubitably shows that a theoretical apparatus can be applied to empirical data in such a way as to produce, in the context of the apparatus, representations of the relevant systems that are of necessity predictively accurate; those representations, moreover, are automatically fruitful in the sense that they are instantly ready to apply to

\footnotetext{
${ }^{37}$ Indeed, structural evidence of the kind relevant to abduction in general differs in important ways from "raven counting" evidence and from HD predictive evidence, primarily because of the non-trivial mathematical relations among theories one must account for in formulating and analyzing the evidence. A general discussion is beyond the scope of this paper.

${ }^{38}$ To get a sense of the difficulty and depth of the technical problems one faces, consider the family of all possible "theories" one can construct in the Newtonian framework, i.e., all (say) twice-differentiable functions (force laws) on Newtonian spacetime. This can not even be turned into a topological manifold, since the different force laws can depend on any number of different variables (position, velocity, fluid flow, shear-stress, and so on), and so are functions with infinitely many different possible domains. Restrict attention, therefore, to the family of all possible theories for a fixed set of physical quantities (say, those appearing in Navier-Stokes theory). This, then, forms an infinite dimensional vector space, modeled on the field of real numbers, which can be turned into a Banach space by choice of a norm; there is, however, no obvious, unique, physically significant choice of norm, though many are available (e.g., the sup-norm, and so on). There is as well no obvious, unique, physically significant topology to put on the space, though many topologies are available (compact-open, Whitney, one based on the sup-norm, and so on). One now wants to put something like a probability measure on it that respects the topology, in the sense that small perturbations don't drastically change the measure of open sets, so one wants a Borel measure that is, if not invariant under linear translations, then uniformly bounded in some way by the size of the translation. There is, strictly speaking, no probability measure on such an infinite-dimensional space, and no translation-invariant Borel measure on it at all (Curiel 2017b). It is an open - and difficult - question whether one can construct such a measure that is uniformly bounded under translations. Even if one were to construct such a measure, it would necessarily assign infinite size to the space as a whole, and so one would need some sort of regularization scheme to extract meaningful probabilities for non-trivial open sets. See Curiel (2017b) for a discussion of how to try to do this in the context of defining physically significant probability measures in cosmology, and the many further problems that arise.

One may be able to get the technical machinery under control if one can "localize" the family of laws to an appropriately restricted subfamily. Consider the case of Newton's and Le Verrier's use of the Precession Theorem to support the inverse-square form of Newton's gravitational law. The "state space" of laws now consists of functions of the form $\frac{1}{r^{2+\epsilon}}$, for small $\epsilon \geq 0$ and, say, $\leq .5$. This naturally forms a compact 1-dimensional manifold with boundary, with a natural, physically significant metric function on it (the absolute difference in $\epsilon$ ). There is thus an obvious, physically significant topology to put on the space, based on the metric. The absolute difference in $\epsilon$ gives the space a locally affine structure: if $\frac{1}{r^{2+\epsilon_{1}}}$ and $\frac{1}{r^{2+\epsilon_{2}}}$ are in the space $\left(\epsilon_{1}<\epsilon_{2}\right)$, then so is $\frac{1}{r^{2+\epsilon^{\prime}}}$ for all $\epsilon^{\prime}=\lambda \epsilon_{1}+(1-\lambda) \epsilon_{2}$, for $\lambda \in[0,1]$. One now wants to put a probability measure on it that respects the topology and the affine structure, in the sense that small perturbations don't drastically change the measure of open sets. One therefore wants a Borel measure that is invariant under affine translations, or at least uniformly bounded in some way by the size of the translation. There are many such measures.
} 
the attempted representation of further systems one has reason to believe are of the same genus. The systematic production of successful theoretical representations of the nature and behavior of physical systems, however, is the most fundamental and most important form of confirmation, whatever form that production may take.

Structural confirmation is a far stronger and more substantial form of confirmation than that provided by merely deducing predictions and matching them against observations, as Maxwell himself emphasized. As he says, "A higher function which [the framework] must discharge is that of presenting to the mind in the clearest and most general form the fundamental principles of dynamical reasoning." This is what gets confirmed - not just the abstract structure of the framework manifest in the phenomena, but all the fundamental principles of dynamical reasoning that are part of the framework and allow one to apply the framework to the experimentally characterized phenomena. Structural confirmation, therefore, provides us more than just good reason to accept the framework as a useful theoretical tool for the scientific investigation of parts of the physical world (or, in more standard parlance, does more than give us good reason to have credence in the framework). Structural confirmation substantiates the fruitfulness of the framework as a system within which one has good reason to believe that one can formulate theories that automatically inherit a high degree of confirmatory support in the standard sense.

The fact that Newtonian abduction directly supports the formulation of and arguments based on subjunctive conditionals provides further confirmatory support of a sort that standard accounts based on HD cannot. It is, again, an instance of the framework's making possible more elaborate and convincing argumentation in favor of a theory's empirical success than HD by itself, or any other form of scientific reasoning standardly treated in accounts of confirmation, makes possible. The production and use of subjunctive conditionals, exemplified by Newton's and Le Verrier's use of the Precession Theorem, is confirmatory of the framework itself, because the derivation of the relevant subjunctive conditionals is possible only by use of the framework itself as whole, not any individual theory in isolation, since the conditionals are comparative among different theories. It is exactly the topological, algebraic, geometric, differential, and other structures that the framework imposes on its family of theories that permits this kind of reasoning. Reasoning of the HD type cannot accomodate this, because it deals with theories in isolation from each other. It has no place for reasoning based on structures accruing to a family of theories. ${ }^{39}$

For the same reasons, confirmation accrues to the framework itself by the further use of such subjunctive reasoning to construct sequences of ever more detailed and accurate individual models, as Newton and Le Verrier did in calculating the orbital perturbations due to inter-planetary gravitational effects, and similarly for Newton's treatment of comets, showing that they all accorded with the predictions of the theory even more accurately than the initial individual models did (Harper 2011; Smith 2014). That the framework supports such successful reasoning, employing the theory itself in powerful ways to directly yield more accurate representations based on abductive arguments, confirms the empirical propriety and adequacy of the framework. I call confirmation based on the formulation and successful use of subjunctive conditionals modal confirmation. I will

\footnotetext{
${ }^{39}$ I do not claim that standard forms of HD reasoning, and standard accounts of confirmation based on them, cannot be modified or extended to cover the form of reasoning I discuss here. I claim only that no account I know of in its present form has the capacity to do so. It would be an interesting project to attempt to modify or extend extant accounts to try to do so.
} 
use confirmation by Newtonian abduction to refer to both structural and modal confirmation, when what I say applies to both.

Similarly, when the predictions of a theory fail and modifications of its individual models cannot mitigate the failure, the framework will provide structures, concepts and methods for guiding us in trying to figure out why it failed, and possibly in trying to formulate new theories by abduction that will provide accurate representations of the concrete models. Newton's framework for theories of light and color, for example, did this in grounding the move from a particle to a wave theory of light at the beginning of the 19th Century. If a framework does so successfully, this also provides confirmation, for it is based on its abductive character: the framework can, in the best cases, tell one what other theories or sorts of theories to look for and examine, and when the search is successful, then confirmation by Newtonian abduction accrues to the framework. This is methodologically richer than HD, and each such success compounds the confirmation accruing to the framework.

Structural confirmation is in itself non-contrastive. Confirmation does not accrue to the framework by being stronger or more appropriate than rivals, and one does not need to worry about "unconceived alternatives" (Stanford 2006): one has shown that the framework does everything one could possibly ask of it; asking more would be to demand the supererogatory. Of course, one is still free to compare the abductive power of different frameworks, when more than one is available in a context in which theories can be abducted possessing individual models appropriate for identification with the same family of concrete models. There are then (at least) three ways that one of the frameworks can garner more confirmation from abductive success than the other. First, one of the frameworks may contain a theory whose individual models apply to the given family of concrete models and to others in addition, which the other framework's theories cannot. Second, the individual models of the theories of one of the frameworks may be predictively more accurate than those of the other. Third, the individual models of one of the frameworks may ground the possibility for novel predictions that the other does not, and those predictions can then be confirmed. All three conditions obtain for general relativity as compared to Newtonian mechanics (in so far as we are able to test separately the predictive accuracy of theories formulated in each framework against the same concrete models, which we cannot in fact always do). ${ }^{40}$ It also follows that, by any measure, a higher degree of confirmation should accrue to a framework that generates more theories that are successful than to one that generates fewer over the same domain of physical systems.

I am not claiming that a physical theory has strong confirmation only in so far as one can cast it

\footnotetext{
${ }^{40} \mathrm{I}$ am glossing over a subtlety here. The concrete models one compares to the individual models of each framework will not always be the same, but will rather sometimes have to be constructed from the raw data in different ways using the different theoretical concepts and structures of the different frameworks, so as to fit into the different abductive propositions of each framework. That is not in general a problem. In such cases, the individual models of one can be "translated" into those of the other in a way that preserves enough physical significance for one to have confidence that the individual models in the different theories represent the same dynamical behaviors of the same physical systems. Such translations often take the form of limiting or approximative constructions. For example, Malament (1986), in effect, shows how to do this for general relativity and Newtonian gravitational theory. If one cannot construct such translations, then one has no reason to believe that the two theories are representing the same behaviors of the same physical systems. Nonetheless, it is sometimes the case that the same concrete models can in fact be identified with individual models of different frameworks, as in the case of Keplerized planetary orbits and individual models in general relativity (Schwarzschild spacetimes with test particles traversing geodesics in a $3+1$ representation of spacetime) and Newtonian mechanics (two-body solutions of Newtonian gravitational theory).
} 
in Lagrangian form, or only in so far as one has an abductive derivation of its equations of motion from a collection of concrete models constructed from experimental or observational results. I quoted Maxwell on the Lagrangian framework and discussed it at length only to drive the point home, as it comes out with such marvelous clarity there: a theory is successful in representing a genus of physical system in the epistemically strongest way, with the highest degree and quality of confirmation, when the structure of the theory's generic equations of motion not only conforms to but embodies the structure manifest in the phenomena it purports to represent, that is, when the structure of the theory recapitulates the structure manifest in the physical evolutions of the system and as little else as possible. That is what the biconditional at the heart of Newtonian abduction guarantees. ${ }^{41}$

One may be concerned that my arguments seem to require that methodological and epistemological principles such as "rules of evidential warrant", "standards of good argumentation", and so on, be such as to admit confirmation, which it seems they must if they are to be included in the framework and the framework as a whole admits confirmation. I claim that it does make sense to treat them as susceptible of confirmation, in the sense that one has reason to use and trust them after they have been shown to play an ineliminable role in successful scientific reasoning. (I say more about this in $\S 5$ below.) One must be careful, however, in one's judgement of what "belongs to a framework" and what does not. When I speak, e.g., of "principles of good argumentation" as forming part of a framework, I do not mean to imply that I think that first-order predicate logic thereby must be part of it (and is thereby susceptible to confirmation). I mean rather: forms of argument peculiar to the domain of physical systems the framework purportedly treats. For some argumentative purposes with regard to a given type of physical system, some "standard" forms of mathematical and heuristic argumentation will not be appropriate, even though they work well in other fields of physics.

An example is how perturbations are treated, when understood as leading terms in a (truncated) power expansion in some dynamically relevant parameter. That works well for systems that are well behaved in an appropriate sense, but not for others, e.g., chaotic systems. Thus, the argument in the 19th Century that the Solar System is stable because it is so up to second order in a standard expansion representing the perturbative effects of the planets' gravitational forces on each other, though unobjectionable in and of itself as a completely standard form of perturbative reasoning, actually fails, because, as Poincaré showed, the third-order terms exhibit chaotic instabilities. The lesson is that one must be extremely careful in applying perturbative analysis to any Newtonian gravitational system of more than two bodies. The recognition and respecting of the required care is part of the canon of "good argumentative forms" for that theory. Another good example is the use of the linearized Einstein field equation as a truncated perturbative expansion. One must be careful in ascertaining that the phenomena at issue are in the appropriate "weak field regime" before one one is justified in using the linearized form in general relativity. Similarly, with regard to standards of what can count as good evidence, only what is peculiar to the framework or theory

\footnotetext{
${ }^{41}$ I believe that Newton's infamous proclamation, "Hypotheses non fingo," is best understood in the context of his characteristically abductive form of reasoning: whatever is not derived abductively from the phenomena is a hypothesis, and those he avoids as much as possible, precisely because, being merely postulated and then tested by weaker forms of reasoning (such as HD or induction), they cannot accrue the same degree of certainty as propositions derived by abduction.
} 
forms a part of it in my sense. An example is stellar aberration. Before telecopic observational prowess, in conjunction with calculational capacity, had developed to such an extent that the phenomenon could be measured and controlled for, worrying about it did not form a part of good evidentiary standards peculiar to astronomy; afterward, it did.

Because it is the only work in the literature I am aware of that more or less directly addresses the issues I investigate here, before concluding this section I will discuss Henderson, Goodman, Tenenbaum, and Woodward (2010). ${ }^{42}$ I think their analysis is interesting and potentially fruitful in many ways, but I will here focus on its shortcomings with regard to the issues I am concerned with.

Their account distinguishes between higher and lower level theoretical systems, which they at different times and in different contexts refer to as 'frameworks', 'theories', and 'hypotheses', for both lower and upper level systems. (Nothing hinges on the differences in terminology.) I shall use 'framework' for a higher-level theoretical system in their account and 'theory' for a lower-level one, which may be generated by a framework. One of the virtues of their account is that they are usefully ambiguous about what "generation" of a theory from a framework may be - it may be deductive entailment, or constructive approximation, or it may instantiate some other relation entirely. Thus nothing prima facie rules out that their analysis may be relevant to Newtonian abduction.

In the account, each level is conceived of as an unstructured set of mutually exclusive alternatives. This, however, from the start misses out on several important features of the relationships among competing frameworks and those among competing theories in a single framework. A framework in general endows its associated family of theories with rich mathematical structures (topological, geometric, algebraic, those grounded in real or complex analysis, and so on), all with manifest physical significance, as was seen in the discussions of the Precession Theorem and modal confirmation. Nothing prevents Henderson, et al., from endowing each level of a hierarchy with such structures, but neither would anything in their account allow one to exploit those structures, because of the strong sense in which they conceive of theories as being mutually exclusive: the relationship of one theory to another, in their setup, cannot tell us anything about the confirmatory support of either theory. This restriction entails that their account cannot represent and account for in an accurate way some of the strongest possible confirmatory evidence for a framework or a theory, viz., modal confirmation arising from the successful use of subjunctive conditionals based on abductive reasoning. I see no way around this problem in their approach. It is important to note, however, that this problem is not peculiar to their account. It infects any account of confirmation in which theories are represented as independent in this strong sense, such as in the Bayesian one (which Henderson, et al., work in).

In any event, it is difficult to see how abductive confirmation of the sort I explicate here can be represented at all in their account. Let $T$ be a lower-level theory, $F$ a higher-level one (a framework)

\footnotetext{
${ }^{42}$ Works such as Lipton (2004, ch. 7) and Henderson (2014) are not relevant to my arguments, as their discussions of abduction treat it as IBE. Lipton does talk about the capacity for IBE to confirm entire frameworks (e.g., on p. 60, in his discussion of Newtonian mechanics and special relativity). His reasons for saying this, however, are based on theoretical - non-empirical - virtues, such as unification, scope and explanatory power, that he claims accrue to frameworks based on their role in IBE. As should be clear, my arguments that Newtonian abduction can confirm frameworks derive directly from the logical form of the reasoning itself in its immediate contact with empirical evidence.
} 
that generates it, and $D$ some structured data that stands in direct evidential relation to $T$. They consider the probabilities $P(T \mid F)$, and $P(F \mid D)$, but they never consider $P(F \mid T)$ or $P(F \mid T \wedge D)$, much less $P(F \mid T \equiv D)$, which represent the fundamental relations I am concerned with. In fact, it is not even clear to me what $P(T \mid F)$ means in their account, since the two theoretical systems live in different probability spaces (since each level of the hierarchy has its own probability space). In so far as one can make sense of $P(T \mid F)$ in their approach, however, then nothing further in their discussion prima facie seems to preclude a discussion of $P(F \mid T), P(F \mid T \wedge D)$, and $P(F \mid T \equiv D)$ in the same vein as theirs, but neither is it clear to me how such an analysis would go, nor how one would justify it in the terms of their arguments, especially in light of the difficulties I pointed out in the previous paragraph (and those I discuss in footnote 38). It would be interesting to investigate whether or not this can be done.

Finally, on their account higher-level frameworks do not gain direct confirmatory support from data, but gain it only indirectly, mediated by the theories they generate that are confirmed by the data (p. 185). This misses out on the fundamental logical structure of Newtonian abduction that lends direct and immediate confirmation to the framework itself from the empirical evidence: the data and the theory enter into the confirmatory relation in a symmetrical, equivalent way (the biconditional component of the abductive proposition), and so the structured data itself in its instantiation of the framework's abstract structure lends the framework confirmatory support as directly as does the success of the theory in producing individual models identifiable with the concrete models in a predictively accurate way à la HD-confirmation.

Nonetheless, I emphasize again that I do not think that Henderson, Goodman, Tenenbaum, and Woodward (2010) has nothing in its favor. To the contrary, I find the approach compelling in many ways and a potentially rich source of ideas and machinery for approaching many important problems in confirmation theory. It is also useful for my particular purposes here: in so far as their analysis is successful, it shows another way that confirmation can accrue to frameworks besides by Newtonian abduction. I wanted only to underscore the differences in aim, in detail of implementation, and in consequence between their work and the present project.

Romeijn (2013) is another interesting case. Although the subject of his arguments is the construction of a Bayesian model of abduction as, in effect, IBE, his analysis is general enough to cover all forms of the generation of "suppositions" from data, and so arguably encompasses at least part of the meat of Newtonian abduction. He says (p. 437),

What the above examples show is that if we happen to choose the theoretical concepts well [by IBE], then they will be beneficial to the predictions, instead of being irrelevant or detrimental. In other words, the examples show that theoretical notions have an evaluative rather than a generative use.

This harmonizes well, in obvious ways, with my discussion of Newtonian abduction, so far as it goes. Newtonian abduction, however, allows one to go further than Romeijn's insightful analysis does: the logical entailment of the biconditional between the theory and the concrete models - this biconditional being the analogue of Romeijn's "choice of theoretical concepts", when the framework is already in place supports confirmation of the framework itself. Romeijn is, I think rightly, reluctant to conclude that his own analysis supports that conclusion.

His analysis also illuminates how Newtonian abduction is superior to IBE in one important 
way. As he observes (p. 437),

[N]othing in [my arguments] answers to the problem [with IBE] that there are infinitely many theoretical notions that are potentially useful [in attempting to model given phenomena], and that we do not have any reason to choose any particular one when learning from the observations.

Within the fixed context of a framework, when Newtonian abductive reasoning is available, this is false: if a framework abducts a successful theory from the phenomena, then one has the strongest possible grounds for choosing the framework, and choice of the framework just is Romeijn's "choice of theoretical concepts". When "choice of theoretical concepts" means choice of theory in a framework rather than choice of a framework itself, Romeijn's pessimism is again unfounded when Newtonian abduction is available, since that picks out the unique theory in the framework best suited to represent the phenomena. When there is more than one framework available for the representation of a given family of concrete models, moreover, and both support the abductive generation of a theory to represent those models, then one may have available the kind of comparative confirmation of the frameworks I discussed above, on the basis of which to choose in a principled way between them. This, by the way, also rebuts the "argument from a bad lot" proposed by van Fraassen (1990), at least in the context of Newtonian abduction, again showing its superiority to standard IBE.

As I mentioned in footnote 15, agreeing determination of the values of parameters by theorymediated measurements, as characterized by Harper (2011) and Smith (2014), is not an HD prediction but rather has the form of a Newtonian abduction. In these cases, different theories are used to determine the value of some physical parameter, and the agreement in values derived from different theories provides strong evidence in favor of that value, at the same time as it provides confirmatory support for the theories themselves. An edifying example is the determination of the value of the cosmological constant $\Lambda$ in the standard $\Lambda$ CDM model of contemporary cosmology, by way of concrete models constructed from observations of: the distribution of the velocities of supernovæ; the spectrum of primordial mass-energy density amplitude-fluctuations encoded in the cosmic microwave background radiation (CMBR); the distribution of anisotropies in the CMBR; the ratio of the mass of x-ray emitting gas in galaxies to total galactic mass; and the observed age of the oldest stars compared to the observed age of the beginning of large-scale structure formation. (See Frieman, Turner, and Huterer 2008.) Each of these observations yields a value for the cosmological constant by reasoning of the form of a Newtonian abduction, each in a different theory. The fact that all such determinations agree manifestly provides substantial confirmation both for the value of the parameter and for the theories themselves. The only structure all these different theories have in common, however, is the framework of general relativity in which all the theories are formulated, by which they are each able to make substantive contact with the $\Lambda$ CDM model in the first place to derive the value of $\Lambda$ from their respective concrete models, and by virtue of which one can identify the $\Lambda$ in each theory as representing the same physical quantity as those in the others. In so far as confirmation accrues to the individual theories based on their role in the reasoning that determines the value of the parameter, so must confirmation accrue to the framework as well, for without the framework one could not even compare the different values in a meaningful way: the shared framework guarantees that the different theories are representing 
the same physical quantity, and so the framework itself plays an ineliminable role in the abductive reasoning resulting in the agreeing measurements.

Finally, as I noted in footnote 14, Newtonian abduction has the form of a Carnapian conditional definition (Carnap 1936, pp. 441ff.), a form of reduction sentence. Carnap, and later philosophers such as Hempel (1955, pp. 23ff.), considered the uses of this logical form only with regard to the definition of theoretical terms in a formal scientific language by reduction in a technical sense to observational terms in that language, not, as here, as the basis for a distinctive form of scientific reasoning. Still, the comparison is illuminating. Hempel (1955, p. 26), for example, says,

A reduction sentence [of the conditional form] offers no complete definition for the term it introduces, but only a partial, or conditional, determination of its meaning; it assigns meaning to the "new" term only for its application to objects which satisfy specific "test conditions."

The "test conditions" Hempel refers to are the analogue of the concrete models appearing in the biconditional of the abductive proposition (3.1). Thus, in a similar vein, one may construe the abductive proposition as the determination of the partial or conditional validity of the theory, and thereby the determination in part of its empirical content and meaning, when the "test conditions" constituted by the phenomena that yielded the concrete model in conjunction with the framework are satisfied. In so far as the framework provides the theoretical basis for the attribution of empirical content to a theory, and in so far as the framework acts, so to speak, as the constitutive a priori component of that empirical content, confirmation of the theory by validation of its empirical content should flow upwards to lend confirmation to the framework as well.

\section{This Is True Confirmation}

When I presented the material of this paper in a talk at the conference "Reasoning in Physics", at the Center for Advanced Studies at Ludwig-Maximilians-Universität in Munich, in December 2016, a sizeable part of the audience objected that Newtonian abduction does not provide real confirmation to frameworks, but rather articulates only an explanatory or grounding relation among frameworks, theories and structured data. First, I contend that, in so far as the abductive proposition implies a proposition having the same logical structure as HD reasoning as used in standard accounts of confirmation, and that the empirical content of the terms in that proposition are essentially the same as in the standard HD case, the presumption must be strong that Newtonian abduction provides confirmation of frameworks at least in that same way, even if in no other way. Second, even if my analysis does articulate an explanatory or grounding relationship, then eo ipso the relationship is confirmatory in the standard sense: to show that a theoretical system explains or grounds the success of our theoretical representations of the world by itself provides confirmatory support for the system in the standard sense (Thešić, Eva, and Hartmann 2017).

As I argued in $\S 4$, however, the type of confirmation that Newtonian abduction confers on frameworks goes beyond that of standard accounts. It provides confirmation by showing how an abductively successful framework can give us understanding of the physical world - we come to see that and how the phenomena in the world of our experience manifest the abstract structure 
of the framework. By "understanding" here, I mean something like "the capacity to operate successfully in the scientific enterprise, in all its forms, aspects and parts"- to use our theoretical representations and experimental practices and results as the basis for the fruitful continuation of the enterprise: as part of evidential warrant in testing; as basis for characterizations of systems and predictions about them; as inspiration for potentially fruitful new investigations; as the grounds for conceptual clarification and innovation in foundational work; and perhaps most of all to grasp how our theoretical representations and reasoning on the one hand and our experimental practices and results on the other relate to, inform, and substantively contribute to the constitution of each other, and to grasp that in such a way as to ground their use in the fruitful continuation of the enterprise. To come to understand the phenomena in this way - to grasp that they manifest the structure of the framework - eo ipso gives one the capacity to do all this, by guiding one's use of the framework in further investigation both of the phenomena one has already investigated and of other systems one has reason to believe are appropriately similar. Deduction of the individual models from equations of motion cannot do this, for it can show at most that the phenomena manifest a structure compatible in some way with that of the equations. It is the entailment of the biconditional between the theory and the concrete models that shows the phenomena to manifest exactly that structure. ${ }^{43}$

Newtonian abduction is superior in this way as well to IBE, viz., with regard to how it provides understanding and explanation. As Romeijn (2013, p. 437) makes clear, it is difficult to see how IBE supports any substantive understanding of why a theory is predictively accurate for a class of phenomena, nor of why the theory explains the phenomena. Newtonian abduction, to the contrary, shows how we may come to a deeper understanding of the physical world even in the complete absence of explanations: Newton developed the theory of universal gravity by explicitly abstaining from giving any "explanation" of the origin of the force, mechanical or otherwise. It would, nonetheless, be folly to deny that the development of that theory did not contribute in a deep and profound way to our understanding of the world.

Much of the empiricist tradition over the past 90 or so years in the study of the relations between data and theory have led to skeptical claims and questions about the need for or possible uses of theory. Hempel (1965), e.g., went so far as to question why theories are necessary at all, given that (as he claimed) learning at the theoretical level always follows learning at the observational one. ${ }^{44}$ More recent philosophers, such as Cartwright (1999), have also wanted to do away with theories, focusing rather on models of various sorts as the fundamental tool or unit of analysis in science based on the idea that the world is too complex and multifarious to be adequately captured by monolithic theories. Newtonian abduction provides an explicit picture that shows why the motivations behind such questions, arguments and conclusions are misguided.

\footnotetext{
${ }^{43}$ I want to emphasize again that none of this has anything to do with any issue pertaining to realism and antirealism. There is no claim made or needed that the structure manifest in the phenomena, i.e., the structured data, is "really" part of the furniture of the world, in some deep metaphysical sense. That it is manifest in the phenomena, in the sense that one can identify the structured data the phenomena yields with individual models, suffices for the soundness of the evidential and confirmatory relations at issue. Those relations are agnostic about realism, as, again, any good confirmatory relation should be.

${ }^{44}$ Stein (1967) provides a compelling confutation of this claim, by showing that, in Newton's development of his abstract framework of dynamics, his metaphysics of space, time and motion, and his theory of universal gravitation, much conceptual and theoretical clarification-learning — was required before the empirical data could be properly comprehended at all, much less used as the basis for the construction of a fully fledged theory.
} 
In the context of the framework, the theory and the concrete model enter into the explanatory relations on an even footing (the biconditional), each providing grounds for improved and deepened understanding of the other. Learning about one happens simultaneously with learning about the other, each informing and informed by the other, inextricably linked.

Much of my discussion of confirmation implicitly rejects an idea that seems to be popular today: that confirmation must be conceived of as giving a reason to think the thing confirmed is true. I, however, do not see why one needs to be a realist in order to talk about and accept the idea (and practice) of confirmation. For me, if one can show that a theoretical structure in conjunction with methodological and epistemological principles, appropriately applied, all conduce, in ineliminable ways, to success in the scientific enterprise, then one has eo ipso endowed them with some measure of confirmatory support. If one then wants to go on to talk about truth, one is welcome to do so, but it is not necessary. In any event, I take it that asserting a theory to be "true" or "approximately true" differs from asserting that the theory's predictions about and descriptions of systems in the world are true or approximately true. One who does not endorse any of the standard forms of scientific realism will avoid the former but can welcome the latter, but it is only the latter that has any relevance to confirmation.

This rejection explains my claim, expressed in $\S 4$, that confirmation by Newtonian abduction, though it lends confirmatory support to Lagrangian mechanics, does not give us a reason to believe that Lagrangian mechanics is true, and indeed that I am not even sure what a truth claim about Lagrangian mechanics could amount to. It is, moreover, not only Lagrangian mechanics that I do not understand bald truth claims about - the same goes for Newtonian mechanics, and for every other framework. Newtonian mechanics is not about any particular kind of physical system, the way that Newtonian gravitational theory is about physical systems with inertial mass, gravitational mass, and appropriate quantities representing spatial position and correlative spatial velocity. I therefore think there is no inconsistency in my being willing to countenance the idea that Newtonian gravitational theory is true (or not), in so far as Newtonian gravitational theory is demonstrably "about" a well defined class of physical systems in the world, even though I am not comfortable with truth claims about Newtonian mechanics itself. Perhaps one would want to say that, in so far as (ex hypothesi) all physical systems are treatable by theories formulated in the context of Newtonian mechanics and so manifest the structure characteristic of Newtonian theories inherited from the abstract structures of the framework (algebraic structures on the family of dynamical solutions, etc.), the framework itself is "true of the entire world". (In that same sense, Lagrangian mechanics could also be said to be true or false.) I would not necessarily be opposed to that. I would ask only that one be clear that the relation of framework to world is radically different than that of theory to world, and so whatever one means in each case by its truth will not necessarily translate straightforwardly to the other. On a related note, some may want to claim that what I argue may be better understood as showing that frameworks are worthy of pursuit (or of being "accepted"), rather than showing that they are confirmed. As a good card-carrying neo-Carnapian, I reject the distinction, at least as posed in such a flat-footed way.

Before concluding, I want to make a few remarks about the possible disconfirmation of frameworks. Many of those who advocate against the idea that frameworks can be confirmed, such as Kuhn, also deny that frameworks can be disconfirmed in principled ways. My explication of Newtonian abduction shows this to be wrong. If one has a framework that has been successful 
in abducting theories to treat given phenomena, and one then shows by the construction of more finely detailed and accurate concrete models that the phenomena in question do not in fact manifest the structure of the framework, then one has disconfirmed the framework, at least with regard to the given phenomena. If one discovers new phenomena that do not manifest the framework's structure from the start, then one has disconfirmed the framework's possible universal validity. Newtonian mechanics, for instance, would be disconfirmed by the discovery of physical systems whose dynamical evolution can be predicted only if one includes derivatives of spatial position of higher order than the first in the initial data. This is incompatible with the structure of Newton's Second Law, which is inconsistent with such initial data. Quantum field theory in its current incarnations (i.e., anything remotely like the framework characterized by the Wightman axioms) would be disconfirmed by discovering a violation of causality in the form of the observation of superluminal propagation of quantum stuff, or by discovering phenomena that violate the Heisenberg Uncertainty Principle. Pace Popper, if a theoretical structure can be disconfirmed in such ways, it can be confirmed in the converse ways: the more phenomena we discover satisfying the Heisenberg Uncertainty Principle, the greater credence we should have in the soundness of the framework of quantum field theory, at least for phenomena in its regime of applicability.

A piece of reasoning that succeeds in applying theoretical apparatus, based on empirical evidence, to the fruitful representation of the world in a way that gives one warrant to accept the theoretical apparatus and continue to use it eo ipso provides the apparatus with confirmatory support. That is what confirmation is. Newtonian abduction does this.

\section{References}

Abbott, B. et al. (LIGO Scientific Collaboration and Virgo Collaboration) (2016, February). Observation of gravitational waves from a binary black hole merger. Physical Review Letters 116(6), 061102. doi:10.1103/PhysRevLett.116.061102. Preprint: arXiv:1602.03837 [grqc].

Bogen, J. and J. Woodward (1988, July). Saving the phenomena. The Philosophical Review $\operatorname{XCVII}(3), 303-352$. doi:10.2307/2185445.

Brown, E. (1904, April). On the degree of accuracy of the new lunar theory and on the final values of the mean motions of the perigee and node. Monthly Notices of the Royal Astronomical Society LXIV(6), 524-534. doi:10.1093/mnras/64.6.524.

Carnap, R. (1936, Oct.). Testability and meaning. I. Philosophy of Science 3(4), 419-471. Part 1 of a two-part article; reprinted, with omissions, in H. Feigl and M. Brodbeck, eds., Readings in the Philosophy of Science, 1953 (New York: Appleton-Century-Crofts, Inc.), pp. 47-92.

Carnap, R. (1956). Empiricism, semantics and ontology. In Meaning and Necessity: A Study in Semantics and Modal Logic (Second ed.)., pp. 205-221. Chicago: The University of Chicago Press. An earlier version was published in Revue Internationale de Philosophie 4(1950):20-40.

Cartwright, N. (1999). The Dappled World: A Study of the Boundaries of Science. Cambridge: Cambridge University Press. 
Curiel, E. (2001). A plea for modesty: Against the current excesses in quantum gravity. Philosophy of Science 68(3), S424-S441. doi:10.1086/392926.

Curiel, E. (2014). Classical mechanics is Lagrangian; it is not Hamiltonian. British Journal for the Philosophy of Science 65(2), 269-321. doi:10.1093/bjps/axs034.

Curiel, E. (2017a). Kinematics, dynamics, and the structure of physical theory. Unpublished manuscript. Preprint: arXiv:1603.02999 [gr-qc]. Most recent draft available at <http:// strangebeautiful.com/papers/curiel-kins-dyns-struc-theory.pdf $>$.

Curiel, E. (2017b). Measure, topology and probabilistic reasoning in cosmology. Preprint: arXiv:1509.01878 [gr-qc]. Most recent draft available at <http://strangebeautiful.com/ papers/curiel-meas-topo-prob-cosmo.pdf $>$.

Curiel, E. (2017c). On the propriety of physical theories as a basis for their semantics. Unpublished manuscript. Most recent draft available at $<$ http://strangebeautiful .com/papers/ curiel-propriety-adequacy-basis-sems . pdf $>$.

Curiel, E. (2019). Schematizing the observer and the epistemic content of theories. Forthcoming in Studies in History and Philosophy of Modern Physics. Preprint: arXiv:1903.02182 [physics.hist-ph].

Earman, J. and W. Salmon (1992). The confirmation of scientific hypotheses. In M. Salmon, J. Earman, C. Glymour, and J. Lennox (Eds.), Introduction to the Philosophy of Science, pp. 42-103. Englewood Cliff, NJ: Prentice Hall.

Fermi, E. (1937). Thermodynamics. New York: Dover Publications, Inc. The 1956 Dover edition is an unabridged, unaltered republication of the 1937 Prentice-Hall edition.

Fowler, R. and E. Guggenheim (1949). Statistical Thermodynamics: A Version of Statistical Mechanics for Students of Physics and Chemistry (Second ed.). Cambridge: Cambridge University Press.

Freundlich, E. (1915). Über die Erklärung der Anomalien im Planeten-System durch die Gravitationswirkung interplanetarer Massen. Astronomische Nachrichten 201(4803), 49-56. doi:10.1002/asna.19152010302

Friedman, M. (2001). The Dynamics of Reason. Stanford, CA: CSLI Publications.

Frieman, J., M. Turner, and D. Huterer (2008, September). Dark energy and the accelerating universe. Annual Review of Astronomy and Astrophysics 46, 385-432. doi:10.1146/annurev.astro.46.060407.145243. Preprint: arXiv:0803.0982 [astro-ph].

Griffiths, J. and J. Podolský (2009). Exact Space-Times in Einstein's General Relativity. Cambridge: Cambridge University Press.

Hall, A. (1894, June). A suggestion in the theory of mercury. The Astronomical Journal XIV(319), 49-51. doi:10.1086/102055.

Harper, W. (1990). Newton's classic deductions from phenomena. PSA: Proceedings of the Biennial Meeting of the Philosophy of Science Association 2, 183-196. Stable URL: <https: //www.jstor.org/stable/193067>. 
Harper, W. (1999). The first six propositions in Newton's argument for universal gravity. The St. John's Review $\operatorname{xLv}(2), 74-93$. In the special issue of the proceedings of the conference "Beyond Hypothesis: Newton's Experimental Philosophy", St. John's College, Annapolis, MD 19-21 March, 1999. Electronic copy available at <http://digitalarchives.sjc.edu/ files/original/5d140db01fbf5fe6562615e2d3713b6e.pdf $>$.

Harper, W. (2011). Isaac Newton's Scientific Method: Turning Data into Evidence about Gravity and Cosmology. Oxford: Oxford University Press.

Hempel, C. (1955). Fundamentals of concept formation in empirical science. In O. Neurath, R. Carnap, and C. Morris (Eds.), Foundations of the Unity of Science: Toward an International Encyclopedia of Unified Science, Volume II, Chapter 7. Chicago: The University of Chicago Press. Formerly entitled International Encyclopedia of Unified Science.

Hempel, C. (1965). The theoretician's dilemma: A study in the logic of theory construction. In Aspects of Scientific Explanation: And Other Essays in the Philosophy of Science, Chapter 8, pp. 173-226. New York: Free Press. Originally published in H. Feigl, et al. (eds.), Concepts, Theories and the Mind-Body Problem, Minnesota Studies in Philosophy of Science Vol. II, Minneapolis: University of Minnesota Press, 1958, pp. 37-98.

Henderson, L. (2014, December). Bayesianism and inference to the best explanation. British Journal for the Philosophy of Science 65(4), 687-715. doi:10.1093/bjps/axt020.

Henderson, L., N. Goodman, J. Tenenbaum, and J. Woodward (2010, April). The structure and dynamics of scientific theories: A hierarchical Bayesian perspective. Philosophy of Science $77(2), 172-200$. doi:10.1086/651319.

Hertz, H. (1884). Über die Bezichungen zwischen den Maxwell'schen electrodynamischen Grundgleichungen und den Grundgleichungen der gegnerischen Electrodynamik. Annalen der Physik und Chemie XxiII, 84-103. Translated D. E. Jones and G. A. Schott as "On the Relations between Maxwell's Fundamental Electromagnetic Equations and the Fundamental Equations of the Opposing Electromagnetics", in H. Hertz Miscellaneous Papers, London:MacMillan and Co., Ltd., ch. XVII, pp. 273-290. doi:10.1002/andp.18842590904.

Kuhn, T. (1996). The Structure of Scientific Revolutions (Third edition ed.). Chicago: University of Chicago Press. The first edition was published in 1962.

Kuipers, T. (2001). Structures in Science: Heuristic Patterns Based on Cognitive Structures; An Advanced Textbook in Neo-Classical Philosophy of Science, Volume 301 of Synthese Library. Dordrecht: Kluwer.

Lakatos, I. (1970). Falsification and the methodology of scientific research programmes. In I. Lakatos and A. Musgrave (Eds.), Criticism and the Growth of Knowledge, pp. 91-196. Cambridge: Cambridge University Press. Reprinted in his Methodology of Scientific Research Programmes: Philosophical Papers, vol. 1, Cambridge: Cambridge University Press, 1980, eds. J. Worrall and G. Currie, ch. 1, pp. 8-101.

Landau, L. and E. Lifschitz (1975). Fluid Mechanics (Second ed.). Oxford: Pergamon Press. An expanded, revised edition of the original 1959 edition. Translated from the Russian by J. Sykes and W. Reid. 
Landau, L. and E. Lifschitz (1980). Statistical Physics, Part 1 (Third ed.). Oxford: Pergamon Press. An expanded, revised edition of the original 1959 edition, by E. Lifschitz and L. Pitaevskii. Translated from the the Russian by J. Sykes and W. Reid.

Laudan, L. (1977). Progress and Its Problems: Towards a Theory of Scientific Growth. Berkeley: University of California Press.

Le Verrier, U. (1845). Mémoire sur les Variations Séculaires des Éléments des Orbites, pour les Sept Planètes Principales, Mercure, Venus, la Terre, Mars, Jupiter, Saturne, Uranus. Paris: Bachelier.

Le Verrier, U. (1859). Lettre de M. Le Verrier à M. Faye sur la théorie de Mercure et sur le mouvement du périhélie de cette planète. Comptes rendus hebdomadaires des séances de l'Académie des sciences (Paris) 49, 379-383.

Lipton, P. (2004). Inference to the Best Explanation (Second ed.). London: Routledge.

Malament, D. (1986). Newtonian gravity, limits, and the geometry of space. In R. Colodny (Ed.), From Quarks to Quasars: Philosophical Problems of Modern Physics, pp. 181-201. Pittsburgh: Pittsburgh University Press.

Malament, D. and S. Zabell (1980, September). Why Gibbs phase averages work-The role of ergodic theory. Philosophy of Science 47(3), 339-349. doi:10.1086/288941.

Maxwell, J. C. (1856). On Faraday's lines of force. See Maxwell (1965b), pp. 155-229. Originally read before the Cambridge Philosophical Society on December 10, 1855, and February 11, 1856, and subsequently published in the Transactions of the Cambridge Philosophical Society, 1864, x, part I, 27-83.

Maxwell, J. C. (1864). A dynamical theory of the electromagnetic field. See Maxwell (1965b), pp. 526-597. Originally published in three parts: Part I, read before the Royal Society on December 8, 1864, and subsequently published in the Royal Society Proceedings, III, 531536. Part II: Philosophical Transactions of the Royal Society, 1865, CLV, 459-512. Part III: Philosophical Magazine, 1865, xxIx, 152-157.

Maxwell, J. C. (1876). On the proof of the equations of motion of a connected system. Proceedings of the Cambridge Philosophical Society II, 292-294. Reprinted in Maxwell 1965, volume II, 308-309.

Maxwell, J. C. (1891). A Treatise on Electricity and Magnetism (3rd ed.). New York: Dover Publications, Inc. In 2 volumes. 1954 facsimile of the edition originally published by Clarendon Press in 1891, edited by J. J. Thomson.

Maxwell, J. C. (1965b). The Scientific Papers of J. C. Maxwell. New York: Dover Publications, Inc. W. Niven (Ed.). Two volumes, published as one.

Newcomb, S. (1895a). The Elements of the Four Inner Planets and the Fundamental Constants of Astronomy. Washington, DC: US Government Printing Office. Supplement to the American Ephemeris and Nautical Almanac for 1897.

Newcomb, S. (1895b). On the principal fundamental constants of astronomy. Astronomical Journal 14, 185-189. doi:10.1086/102176. 
Newcomb, S. (1905). A New Determination of the Precessional Constant with the Resulting Precessional Motions. Washington, DC: U.S. Nautical Almanac Office.

Newcomb, S. (1911). Gravitation. In H. Chisholm (Ed.), The Encyclopcedia Britannica: A Dictionary of Arts, Sciences, Literature and General Information (Eleventh edition ed.), Volume XII, pp. 384-389. Cambridge: University of Cambridge Press.

Newton, I. (1672). Letter of february 6, 1671/72, to Henry Oldenburg, Secretary of the Royal Society, outlining Newton's researches on light and color. In I. Cohen (Ed.), Isaac Newton's Papers $\&$ Letters on Natural Philosophy, pp. 47-59. Cambridge, MA: Harvard University Press. The original was published in the Philosophical Transactions of the Royal Society, 80(February 16, 1671/72):3075-3087.

Newton, I. (1726). Philosophice Naturalis Principia Mathematica (Third ed.). Amherst, NY: Prometheus Press. The translation by A. Motte of the third edition (1726), originally produced in 1729, and reprinted by Prometheus Press in 1995. The first edition of the Principia was published in 1686, the second in 1713 .

Norton, J. (2005). A little survey on induction. In P. Achinstein (Ed.), Scientific Evidence: Philosophical Theories and Applications, pp. 9-34. Baltimore: John Hopkins University Press.

Peirce, C. S. (1878a). Deduction, induction and hypothesis. See Peirce (1992), Chapter 12, pp. 186-199. Originally published in Popular Science Monthly 13(August, 1878):470-482.

Peirce, C. S. (1878b). The doctrine of chances. See Peirce (1992), Chapter 9, pp. 142-154. Originally published in Popular Science Monthly 12(March, 1878):604-615.

Peirce, C. S. (1903). The nature of meaning. In The Essential Peirce: Selected Philosophical Writings, Volume 2 (1893-1913), Chapter 15, pp. 208-225. Bloomington, IN: Indiana University Press. Originally delivered 07 May 1903 at Harvard University, as the sixth in a series of seven public lectures on pragmatism.

Peirce, C. S. (1955). Abduction and induction. In Philosophical Writings of Peirce, Chapter 11, pp. 150-156. New York: Dover Publications, Inc. Collated by the editor from 4 different manuscripts, 3 of them unpublished.

Peirce, C. S. (1992). The Essential Peirce: Selected Philosophical Writings, Volume 1 (18671893). Bloomington, IN: Indiana University Press. Edited by N. Houser and C. Kloesel.

Romeijn, J.-W. (2013, December). Abducted by bayesians? Journal of Applied Logic 11(4), 430-439. Part of special issue "Combining Probability and Logic: Papers from Progic 2011", ed. J. Helzner. doi:10.1016/j.jal.2012.09.003.

Sakurai, J. (1964). Invariance Principles and Elementary Particles. Princeton: Princeton University Press.

Smith, G. (2014). Closing the loop: Testing Newtonian gravity then and now. In Z. Biener and E. Schliesser (Eds.), Newton and Empiricism, Chapter 10, pp. 262-351. Oxford: Oxford University Press. doi:10.1093/acprof:oso/9780199337095.003.0011.

Sommerfeld, A. (1964). Thermodynamics and Statistical Mechanics, Volume V of Lectures on Theoretical Physics. New York: Academic Press. Trans. J. Kestin. Edited and posthumously completed by F. Bopp and J. Meixner. 
Sorkin, R. (1979, May). On the meaning of the canonical ensemble. International Journal of Theoretical Physics 18(5), 309-321. doi:10.1007/BF00670427.

Stanford, P. (2006). Exceeding Our Grasp: Science, History, and the Problem of Unconceived Alternatives. Oxford: Oxford University Press. doi:10.1093/0195174089.001.0001.

Stein, H. (1967). Newtonian space-time. Texas Quarterly 10, 174-200.

Stein, H. (1989, June). Yes, but...: Some skeptical remarks on realism and anti-realism. Dialectica 43(1-2), 47-65. doi:10.1111/j.1746-8361.1989.tb00930.x.

Stein, H. (1990). "From the phænomena of motions to the forces of nature": Hypothesis or deduction? PSA: Proceedings of the Biennial Meeting of the Philosophy of Science Association 2, 209-222. Stable URL: <http://www.jstor.org/stable/193069>.

Stein, H. (1992). Was Carnap entirely wrong, after all? Synthese 93, 275-295. doi:10.1007/BF00869429.

Stein, H. (1994). Some reflections on the structure of our knowledge in physics. In D. Prawitz, B. Skyrms, and D. Westerståhl (Eds.), Logic, Metholodogy and Philosophy of Science, Proceedings of the Ninth International Congress of Logic, Methodology and Philosophy of Science, pp. 633-655. New York: Elsevier Science B.V.

Stein, H. (unpublished-a). Further considerations on Newton's method. Unpublished manuscript. Available at $<$ http://strangebeautiful.com/phil-phys.html $>$.

Stein, H. (unpublished-b). On metaphysics and method in Newton. Unpublished manuscript. Available at $<$ http://strangebeautiful.com/phil-phys.html $>$.

Szekeres, P. (1975). A class of inhomogeneous cosmological models. Communications in Mathematical Physics 41(1), 55-64. Open-access text available at: http://projecteuclid.org/ euclid.cmp/1103860587. 10.1007/BF01608547.

Thešić, M., B. Eva, and S. Hartmann (2017). Confirmation by explanation: A Bayesian justification of IBE. Unpublished manuscript. Preprint: <http://philsci-archive.pitt.edu/ $13328 />$.

van Fraassen, B. (1990). Laws and Symmetry. Oxford: Oxford University Press.

Wald, R. (1984). General Relativity. Chicago: University of Chicago Press.

Weinberg, S. (2008). Cosmology. Oxford: Oxford University Press.

Wilson, C. (2004). Newton and celestial mechanics. In I. Cohen and G. Smith (Eds.), The Cambridge Companion to Newton, Chapter 6, pp. 202-226. Cambridge: Cambridge University Press.

Worrall, J. (2000). The scope, limits, and distinctiveness of the method of 'deduction from the phenomena': Some lessons from Newton's 'demonstrations' in optics. British Journal for the Philosophy of Science 51, 45-80. 10.1093/bjps/51.1.45.

Yunes, N. and F. Pretorius (2009, December). Fundamental theoretical bias in gravitational wave astrophysics and the parametrized post-Einsteinian framework. Physical Review D 80(12), 122003. doi:10.1103/PhysRevD.80.122003. 